\title{
TITLE:
}

\section{Linear stability of the cylindrical couette flow of a rarefied gas}

$\operatorname{AUTHOR}(S)$ :

Yoshida, H; Aoki, K

\section{CITATION:}

Yoshida, $\mathrm{H}$... [et al]. Linear stability of the cylindrical Couette flow of a rarefied gas. PHYSICAL REVIEW E 2006, 73(2): 021201.

\section{ISSUE DATE:}

2006-02

URL:

http://hdl.handle.net/2433/39913

RIGHT:

Copyright 2006 American Physical Society 
PHYSICAL REVIEW E 73, 021201 (2006)

\title{
Linear stability of the cylindrical Couette flow of a rarefied gas
}

\author{
Hiroaki Yoshida \\ Department of Aeronautics and Astronautics and Advanced Research Institute of Fluid Science and Engineering, \\ Graduate School of Engineering, Kyoto University, Kyoto 606-8501, Japan \\ Kazuo Aoki* \\ Department of Mechanical Engineering and Science and Advanced Research Institute of Fluid Science and Engineering, \\ Graduate School of Engineering, Kyoto University, Kyoto 606-8501, Japan
}

(Received 29 August 2005; revised manuscript received 30 November 2005; published 22 February 2006)

\begin{abstract}
A rarefied gas between two coaxial circular cylinders of infinite length, rotating with different angular velocities and kept at a common temperature, is considered. The stability of the circumferentially as well as axially uniform flow (cylindrical Couette flow) for circumferentially uniform small disturbances is investigated on the basis of kinetic theory. The linear-stability analysis is performed using the Bhatnagar-Gross-Krook model of the Boltzmann equation and the diffuse reflection condition on the cylinders. The maximum growth rate of the disturbances is determined numerically by solving the initial and boundary value problem for the disturbances for relatively small Knudsen numbers and wide ranges of angular velocities of the cylinders. As a result, the parameter range where the cylindrical Couette flow is unstable is clarified. The result is compared with the corresponding result based on the continuum model of the compressible Navier-Stokes type. A comparison is also made with the result of a direct numerical analysis of the original Boltzmann system, obtained by the direct simulation Monte Carlo method in previous papers as well as in the present study.
\end{abstract}

DOI: 10.1103/PhysRevE.73.021201 PACS number(s): 47.45. $-\mathrm{n}, 51.10 .+\mathrm{y}, 47.20 .-\mathrm{k}, 47.40 .-\mathrm{x}$

\section{INTRODUCTION}

Axially and circumferentially uniform flow between two rotating coaxial circular cylinders is a textbook example known as cylindrical Couette flow in classical (incompressible) fluid dynamics. It is also a basic flow for a rarefied gas and has been investigated on the basis of kinetic theory [1-15]. The interest of the existing work extends to various aspects, such as the validation of the generalized hydrodynamics $[3,4]$, the validity of the principle of frame indifference [6], the ghost effect caused by infinitesimal evaporation and condensation on the cylinders [7], bifurcation of flows when evaporation and condensation on the cylinders take place $[9-11,14]$, and inverted velocity profiles in the case of small accommodation coefficients $[8,15,16]$.

On the other hand, the instability of cylindrical Couette flow and the transition to a secondary vortical flow, known as the Taylor-vortex flow, is one of the fundamental problems in incompressible fluid dynamics and have a long history of investigation [17-22]. In contrast, the study of the same problems for a rarefied gas was started rather recently, and some numerical results based on the direct simulation Monte Carlo (DSMC) method [23,24] have been reported [14,24-30]. However, as discussed in Ref. [14], there may be a limitation of applicability of the DSMC method to instability problems. A different approach is taken in Ref. [31], where a precise analysis of the bifurcation of cylindrical Couette flow is performed on the basis of a fluid-dynamic system that is valid for small Knudsen numbers as well as slow rotation but for an arbitrary temperature difference be-

*Electronic address: aoki@aero.mbox.media.kyoto-u.ac.jp tween the cylinders. The attention is focused on the ghost effect $[32,33]$ on the temperature field in the continuum limit caused by the Taylor-vortex flow with infinitesimal flow speed.

In the present study, we investigate the stability of the cylindrical Couette flow of a rarefied gas for small disturbances (linear stability) on the basis of kinetic theory and clarify the onset of the instability without restricting the Mach number and the Knudsen number. In classical fluid dynamics, the standard way for linear-stability analysis is to derive an eigenvalue problem whose eigenvalues determine the temporal growth rates of small disturbances and to solve it numerically by appropriate discretization. In kinetic theory, however, the corresponding eigenvalue problem is much more complicated because of many independent variables and the presence of complicated integrals originating from the basic equation (Boltzmann equation). This fact still applies even if a simplified model equation, such as the Bhatnagar-Gross-Krook (BGK) equation [34,35], is used instead of the original Boltzmann equation. Therefore, we take a different way to bypass the direct solution of the eigenvalue problem. That is, we solve the initial and boundary value problem for a small disturbance numerically with a finite-difference method to obtain the time evolution of the disturbance, from which we deduce the growth rate of the disturbance. From these results, we obtain the so-called neutral stability curves. In order to make the numerical analysis feasible, we adopt the BGK equation as the basic equation.

In addition to the linear-stability analysis mentioned above, we also carry out a direct numerical analysis of the flow between the cylinders by means of the DSMC method for the original Boltzmann system (for hard-sphere molecules) to supplement the results obtained in previous papers $[29,30]$. The parameter range in which the Taylor-vortex flow 


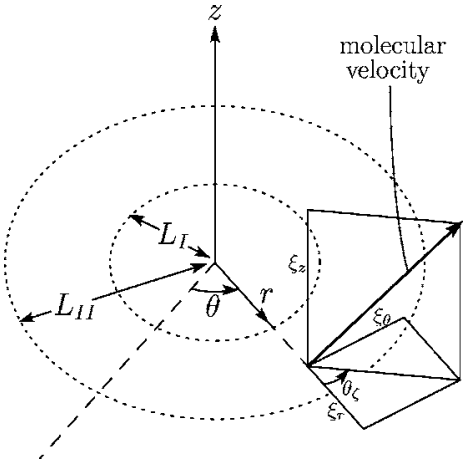

FIG. 1. Coordinate systems.

is obtained by the DSMC method is compared with the result by linear-stability analysis.

\section{PROBLEM AND BASIC EQUATION}

Let us consider a rarefied gas between two coaxial circular cylinders with infinite length. To describe the problem, we use the cylindrical coordinate system $(r, \theta, z)$ with the $z$ axis taken along the common axis of the cylinders (Fig. 1). The radius of the inner cylinder is $L_{I}$ and that of the outer cylinder is $L_{I I}$. The inner and outer cylinders are rotating at constant surface velocities $V_{I}$ and $V_{I I}$ in the $\theta$ direction and kept at a common temperature $T_{0}$. We investigate the behavior of the gas on the basis of kinetic theory under the following assumptions: (i) the behavior of the gas is described by the BGK model equation [34-36]; (ii) the gas molecules undergo diffuse reflection on the surface of the cylinders $(r$ $=L_{I}$ and $\left.L_{I I}\right)$, that is, the reflected molecules are distributed according to the corresponding part of the Maxwellian distribution with the velocity and temperature of the surface, and there is no net mass flow across the surface; (iii) the flow field is axisymmetric, i.e., circumferentially uniform.

This is the basic setting for the linear-stability analysis that is the main subject of the present paper. We will also perform the direct numerical simulation of the gas flow using the DSMC method. For this, we will make a slight modification of the setting, which will be specified in Sec. IV.

We introduce some notation: $t$ is the time; $\boldsymbol{\xi}$ is the molecular velocity with $\xi_{r}, \xi_{\theta}$, and $\xi_{z}$ its $r, \theta$, and $z$ components (Fig. 1$) ; f(t, r, z, \boldsymbol{\xi})$ is the velocity distribution function of the gas molecules; $\rho$ is the density, $\boldsymbol{v}$ the flow velocity with $v_{r}$, $v_{\theta}$, and $v_{z}$ its $r, \theta$, and $z$ components, and $T$ the temperature of the gas; and $R$ is the gas constant per unit mass, i.e., $R$ $=k / m$, where $k$ is the Boltzmann constant and $m$ is the mass of a molecule.

The BGK equation in the present coordinate system is given as

$$
\begin{gathered}
\frac{\partial f}{\partial t}+\xi_{r} \frac{\partial f}{\partial r}+\xi_{z} \frac{\partial f}{\partial z}+\frac{\xi_{\theta}^{2}}{r} \frac{\partial f}{\partial \xi_{r}}-\frac{\xi_{r} \xi_{\theta}}{r} \frac{\partial f}{\partial \xi_{\theta}}=A_{c} \rho\left(f_{e}-f\right), \\
f_{e}=\frac{\rho}{(2 \pi R T)^{3 / 2}} \exp \left(-\frac{(\boldsymbol{\xi}-\boldsymbol{v})^{2}}{2 R T}\right),
\end{gathered}
$$

$$
\begin{gathered}
\rho=\int f d \boldsymbol{\xi}, \\
\boldsymbol{v}=\frac{1}{\rho} \int \boldsymbol{\xi} f d \boldsymbol{\xi}, \\
T=\frac{1}{3 R \rho} \int(\boldsymbol{\xi}-\boldsymbol{v})^{2} f d \boldsymbol{\xi},
\end{gathered}
$$

where $A_{c}$ is a constant $\left(A_{c} \rho\right.$ is the collision frequency of a gas molecule, which is independent of the molecular velocity for the BGK equation), $d \boldsymbol{\xi}=d \xi_{r} d \xi_{\theta} d \xi_{z}$, and the integration with respect to $\boldsymbol{\xi}$ is carried out over its whole space.

The boundary conditions (diffuse reflection) on the cylinders are written as follows: at $r=L_{I}$, for $\xi_{r}>0$,

$$
\begin{aligned}
f\left(t, L_{I}, z, \boldsymbol{\xi}\right) & =\frac{\rho_{w I}}{\left(2 \pi R T_{0}\right)^{3 / 2}} \exp \left(-\frac{\xi_{r}^{2}+\left(\xi_{\theta}-V_{I}\right)^{2}+\xi_{z}^{2}}{2 R T_{0}}\right), \\
\rho_{w I} & =-\left(\frac{2 \pi}{R T_{0}}\right)^{1 / 2} \int_{\xi_{r}<0} \xi_{r} f\left(t, L_{I}, z, \boldsymbol{\xi}\right) d \boldsymbol{\xi},
\end{aligned}
$$

and at $r=L_{I I}$, for $\xi_{r}<0$,

$$
\begin{aligned}
f\left(t, L_{I I}, z, \xi\right) & =\frac{\rho_{w I I}}{\left(2 \pi R T_{0}\right)^{3 / 2}} \exp \left(-\frac{\xi_{r}^{2}+\left(\xi_{\theta}-V_{I I}\right)^{2}+\xi_{z}^{2}}{2 R T_{0}}\right), \\
\rho_{w I I} & =\left(\frac{2 \pi}{R T_{0}}\right)^{1 / 2} \int_{\xi_{r}>0} \xi_{r} f\left(t, L_{I I}, z, \xi\right) d \xi .
\end{aligned}
$$

For convenience of analysis, we introduce dimensionless variables by the following relations:

$$
\begin{gathered}
t=L_{I}\left(2 R T_{0}\right)^{-1 / 2} \hat{t}, \quad r=L_{I} \hat{r}, \quad z=L_{I} \hat{z}, \\
\left(\xi_{r}, \xi_{\theta}, \xi_{z}\right)=\left(2 R T_{0}\right)^{1 / 2}\left(\zeta_{r}, \zeta_{\theta}, \zeta_{z}\right), \\
\zeta_{r}=\zeta \cos \theta_{\zeta}, \quad \zeta_{\theta}=\zeta \sin \theta_{\zeta}, \\
f=\frac{\rho_{0}}{\left(2 R T_{0}\right)^{3 / 2}} \hat{f}, \quad \rho=\rho_{0} \hat{\rho}, \quad T=T_{0} \hat{T}, \\
\left(v_{r}, v_{\theta}, v_{z}\right)=\left(2 R T_{0}\right)^{1 / 2}\left(\hat{v}_{r}, \hat{v}_{\theta}, \hat{v}_{z}\right), \\
V_{I}=\left(2 R T_{0}\right)^{1 / 2} \hat{V}_{I}, \quad V_{I I}=\left(2 R T_{0}\right)^{1 / 2} \hat{V}_{I I},
\end{gathered}
$$

where $\left(\zeta, \theta_{\zeta}, \zeta_{z}\right)\left(0 \leqslant \zeta<\infty,-\pi \leqslant \theta_{\zeta}<\pi,-\infty<\zeta_{z}<\infty\right)$ is the cylindrical coordinate system in the dimensionless molecular velocity space $\zeta=\xi /\left(2 R T_{0}\right)^{1 / 2}$ with the baseline $\left(\theta_{\zeta}=0\right)$ in the $\zeta_{r}$ (or $\xi_{r}$ ) direction (Fig. 1), and $\rho_{0}$ is a reference density, which will be specified in the following section. Then the BGK equation in dimensionless form reads 


$$
\begin{aligned}
\frac{\partial \hat{f}}{\partial \hat{t}}+\zeta \cos \theta_{\zeta} \frac{\partial \hat{f}}{\partial \hat{r}}-\frac{\zeta \sin \theta_{\zeta}}{\hat{r}} \frac{\partial \hat{f}}{\partial \theta_{\zeta}}+\zeta_{z} \frac{\partial \hat{f}}{\partial \hat{z}}=\frac{2}{\sqrt{\pi} \mathrm{Kn}} \hat{\rho}\left(\hat{f}_{e}-\hat{f}\right), \\
\hat{f}_{e}=\frac{\hat{\rho}}{(\pi \hat{T})^{3 / 2}} \exp \left(-\frac{\left(\zeta_{z}-\hat{v}_{z}\right)^{2}}{\hat{T}}\right) \\
\quad \times \exp \left(-\frac{\zeta^{2}-2 \hat{v}_{r} \zeta \cos \theta_{\zeta}-2 \hat{v}_{\theta} \zeta \sin \theta_{\zeta}+\hat{v}_{r}^{2}+\hat{v}_{\theta}^{2}}{\hat{T}}\right),
\end{aligned}
$$

$$
\hat{\rho}=\int \zeta \hat{f} d \zeta d \theta_{\zeta} d \zeta_{z}
$$$$
\hat{v}_{r}=\frac{1}{\hat{\rho}} \int \zeta^{2} \cos \theta_{\xi} \hat{f} d \zeta d \theta_{\zeta} d \zeta_{z}
$$$$
\hat{v}_{\theta}=\frac{1}{\hat{\rho}} \int \zeta^{2} \sin \theta_{\xi} \hat{f} d \zeta d \theta_{\zeta} d \zeta_{z}
$$$$
\hat{v}_{z}=\frac{1}{\hat{\rho}} \int \zeta \zeta_{z} \hat{f} d \zeta d \theta_{\zeta} d \zeta_{z}
$$

$$
\hat{T}=\frac{2}{3 \hat{\rho}} \int \zeta\left(\zeta^{2}+\zeta_{z}^{2}\right) \hat{f} d \zeta d \theta_{\zeta} d \zeta_{z}-\frac{2}{3}\left(\hat{v}_{r}^{2}+\hat{v}_{\theta}^{2}+\hat{v}_{z}^{2}\right)
$$

$$
\mathrm{Kn}=\frac{\ell_{0}}{L_{I}}, \quad \ell_{0}=\frac{2}{\sqrt{\pi}} \frac{\left(2 R T_{0}\right)^{1 / 2}}{A_{c} \rho_{0}},
$$

where $\mathrm{Kn}$ is the Knudsen number, and $\ell_{0}$ is the mean free path of the gas molecules in the equilibrium state at rest at density $\rho_{0}$ and temperature $T_{0}$. Here and in what follows, the integration with respect to $\zeta, \theta_{\zeta}$, and $\zeta_{z}$ is carried out over the domain $\left(0 \leqslant \zeta<\infty,-\pi \leqslant \theta_{\zeta}<\pi,-\infty<\zeta_{z}<\infty\right)$, unless otherwise stated. It is noted that Eq. (7) contains only the $\theta_{\zeta}$ derivative concerning the molecular velocity.

The boundary condition on the inner cylinder is

$$
\hat{f}(\hat{t}, 1, \hat{z}, \zeta)=\frac{\hat{\rho}_{w I}}{\pi^{3 / 2}} \exp \left(-\zeta^{2}+2 \hat{V}_{I} \zeta \sin \theta_{\zeta}-\zeta_{z}^{2}-\hat{V}_{I}^{2}\right),
$$

$\hat{\rho}_{w I}=-2 \sqrt{\pi} \int_{-\pi \leqslant \theta_{\zeta}<-\pi / 2, \pi / 2<\theta_{\zeta}<\pi} \zeta^{2} \cos \theta_{\zeta} \hat{f}(\hat{t}, 1, \hat{z}, \zeta) d \zeta d \theta_{\zeta} d \zeta_{z}$

at $\hat{r}=1$ for $\left|\theta_{\zeta}\right|<\pi / 2$, and that on the outer cylinder is

$$
\hat{f}\left(\hat{t}, L_{I I} / L_{I}, \hat{z}, \zeta\right)=\frac{\hat{\rho}_{w I I}}{\pi^{3 / 2}} \exp \left(-\zeta^{2}+2 \hat{V}_{I I} \zeta \sin \theta_{\zeta}-\zeta_{z}^{2}-\hat{V}_{I I}^{2}\right),
$$

$$
\hat{\rho}_{w I I}=2 \sqrt{\pi} \int_{\left|\theta_{\zeta}\right|<\pi / 2} \zeta^{2} \cos \theta_{\zeta} \hat{f}\left(\hat{t}, L_{I I} / L_{I}, \hat{z}, \zeta\right) d \zeta d \theta_{\zeta} d \zeta_{z}
$$

at $\hat{r}=L_{I I} / L_{I}$ for $-\pi \leqslant \theta_{\zeta}<-\pi / 2$ and $\pi / 2<\theta_{\zeta}<\pi$.

Now the problem is seen to contain the four dimensionless parameters $\mathrm{Kn}, \hat{V}_{I}, \hat{V}_{I I}$, and $L_{I I} / L_{I}$.

We conclude this section by giving a short remark on the BGK model. This model is based on the Boltzmann equation for pseudo-Maxwellian molecules (i.e., the molecules interacting with the intermolecular force inversely proportional to the fifth power of the distance between the molecules and with an angular cutoff) [36]. In this case, the loss term of the Boltzmann equation reduces to the form of the loss term of the BGK equation, i.e., $A_{c} \rho f$. On the other hand, if one assumes that the molecules after collision are distributed according to the local equilibrium distribution at the point under consideration, then the gain term of the Boltzmann equation reduces to the form of the gain term of the BGK equation, i.e., $A_{c} \rho f_{e}$. Thus, the nonlinearity of the gain term is stronger than the original quadratic nonlinearity of the Boltzmann equation though it is preserved in the loss term. The model satisfies the fundamental properties of the Boltzmann equation including the $H$ theorem. It also gives fluiddynamic type systems that are of the same form as those provided by the Boltzmann equation when the Knudsen number is small, and the quantitative difference arises only in the transport and slip coefficients [32]. For instance, the Prandtl number $\operatorname{Pr}=5 R \mu / 2 \lambda$, where $\mu$ is the viscosity and $\lambda$ is the thermal conductivity, is unity for the BGK model but close to 2/3 (2/3 for the pseudo-Maxwellian molecules and 0.6607 for hard-sphere molecules) for the Boltzmann equation. Although this drawback is well recognized, the simplicity of the BGK equation is highly attractive as a tool for preliminary analysis and computation. It also enables us to obtain information about problems that are not tractable by the original Boltzmann equation. In fact, it has been used in such a way and has contributed to the understanding of the phenomena described by the Boltzmann equation.

\section{LINEAR-STABILITY ANALYSIS OF CYLINDRICAL COUETTE FLOW}

\section{A. Basic system for stability analysis}

We first consider the steady $(\partial / \partial \hat{t}=0)$ and axially uniform $(\partial / \partial \hat{z}=0)$ solution, which is known as cylindrical Couette flow, of Eqs. (7), (11), and (12) (recall that we have assumed axial symmetry of the flow). In contrast to the case of a classical incompressible fluid, analytical solution is not available for Eqs. (7), (11), and (12). Therefore, we need to obtain accurate numerical solutions. Let us denote the numerical solution of the cylindrical Couette flow by $f_{C}$ $=\rho_{0}\left(2 R T_{0}\right)^{-3 / 2} \hat{f}_{C}$. We take the average density of $f_{C}$ between the cylinders as the reference density $\rho_{0}$. Then the density $\hat{\rho}_{C}$ corresponding to $\hat{f}_{C}$, i.e., Eq. (9a) with $\hat{f}=\hat{f}_{C}$, is normalized as 


$$
\frac{2}{\left(L_{I I} / L_{I}\right)^{2}-1} \int_{1}^{L_{I I} / L_{I}} \hat{r} \hat{\rho}_{C} d \hat{r}=1
$$

The numerical solution $\hat{f}_{C}$ will be presented in Sec. III B 1 .

Next, we investigate the stability of the cylindrical Couette flow for a small disturbance on the basis of the numerical solution $\hat{f}_{C}$. We assume that the disturbance does not depend on $\theta$ and let the solution $\hat{f}$ be in the following form:

$$
\hat{f}=\hat{f}_{C}+\epsilon \tilde{\mathcal{F}}\left(\hat{t}, \hat{r}, \zeta, \theta_{\zeta}, \zeta_{z} ; \alpha\right) \exp (i \alpha \hat{z}),
$$

where $\epsilon$ is a small parameter $(0<\epsilon \ll 1), \widetilde{\mathcal{F}}$ is a complexvalued function, $\alpha$ is an arbitrary real number, and $i$ is the imaginary unit. To be more precise, the disturbance in Eq. (14) should be the real part of $\epsilon \widetilde{\mathcal{F}} \exp (i \alpha \hat{z})$, but explicit mention is omitted by convention. An arbitrary small disturbance can be expressed by the superposition of $\epsilon \widetilde{\mathcal{F}} \exp (i \alpha \hat{z})$; for instance, a periodic disturbance with period $2 L_{I}$ in $z$ is expressed by the form $\epsilon \sum_{n=-\infty}^{\infty} \hat{\mathcal{F}}_{n} \exp (\pi i n \hat{z}) . \rho_{0}$ is also the average density of the dimensional counterpart of $\hat{f}$ of Eq. (14) over the domain $\left(L_{I}<r<L_{I I}, 0<z<2 \pi L_{I} / \alpha\right)$ because of the periodicity of the disturbance in $z$.

If we insert Eq. (14) in Eq. (7) as well as in the boundary conditions (11a), (11b), (12a), and (12b) and neglect the second and higher powers of $\epsilon$, then we obtain the linearized time-dependent boundary value problem for $\widetilde{\mathcal{F}}$, which can be expressed in the following form:

$$
\begin{gathered}
\frac{\partial \tilde{\mathcal{F}}}{\partial \hat{t}}=\mathcal{L}[\tilde{\mathcal{F}}], \\
\tilde{\mathcal{F}}=\mathcal{L}_{B I}[\tilde{\mathcal{F}}] \quad \text { at } \hat{r}=1 \text { for }\left|\theta_{\zeta}\right|<\pi / 2,
\end{gathered}
$$

$$
\begin{aligned}
\tilde{\mathcal{F}}= & \mathcal{L}_{B I I}[\tilde{\mathcal{F}}] \\
& \text { at } \hat{r}=L_{I I} / L_{I} \text { for }-\pi \leqslant \theta_{\zeta}<-\pi / 2 \text { and } \pi / 2<\theta_{\zeta}<\pi .
\end{aligned}
$$

Here, $\mathcal{L}$ is the integro-differential operator defined by

$$
\begin{aligned}
& \mathcal{L}[\tilde{\mathcal{F}}]=\frac{2}{\sqrt{\pi} \mathrm{Kn}}\left[\tilde{\mathcal{D}}\left(\hat{f}_{e C}-\hat{f}_{C}\right)+\hat{\rho}_{C}\left(\tilde{\mathcal{F}}_{e}-\tilde{\mathcal{F}}\right)\right] \\
& -\left(\zeta \cos \theta_{\zeta} \frac{\partial}{\partial \hat{r}}-\frac{\zeta \sin \theta_{\zeta}}{\hat{r}} \frac{\partial}{\partial \theta_{\zeta}}+i \alpha \zeta_{z}\right) \tilde{\mathcal{F}}, \\
& \tilde{\mathcal{F}}_{e}=\hat{f}_{e C}\left[\frac{\tilde{\mathcal{D}}}{\hat{\rho}_{C}}+\frac{2\left[\zeta \cos \theta_{\zeta} \tilde{\mathcal{V}}_{r}+\left(\zeta \sin \theta_{\zeta}-\hat{v}_{\theta C}\right) \tilde{\mathcal{V}}_{\theta}+\zeta_{z} \tilde{\mathcal{V}}_{z}\right]}{\hat{T}_{C}}\right. \\
& \left.+\left(\frac{\zeta^{2}-2 \hat{v}_{\theta C} \zeta \sin \theta_{\zeta}+\zeta_{z}^{2}+\hat{v}_{\theta C}^{2}}{\hat{T}_{C}}-\frac{3}{2}\right) \frac{\tilde{\mathcal{T}}}{\hat{T}_{C}}\right], \\
& \widetilde{\mathcal{D}}=\int \zeta \widetilde{\mathcal{F}} d \zeta d \theta_{\zeta} d \zeta_{z}
\end{aligned}
$$

$$
\begin{gathered}
\tilde{\mathcal{V}}_{r}=\frac{1}{\hat{\rho}_{C}} \int \zeta^{2} \cos \theta_{\zeta} \tilde{\mathcal{F}} d \zeta d \theta_{\zeta} d \zeta_{z}, \\
\tilde{\mathcal{V}}_{\theta}=\frac{1}{\hat{\rho}_{C}} \int \zeta\left(\zeta \sin \theta_{\zeta}-\hat{v}_{\theta C}\right) \tilde{\mathcal{F}} d \zeta d \theta_{\zeta} d \zeta_{z}, \\
\tilde{\mathcal{V}}_{z}=\frac{1}{\hat{\rho}_{C}} \int \zeta \zeta_{z} \tilde{\mathcal{F}} d \zeta d \theta_{\zeta} d \zeta_{z}, \\
\tilde{\mathcal{T}}=\frac{2}{3 \hat{\rho}_{C}} \int \zeta\left(\zeta^{2}-2 \hat{v}_{\theta C} \zeta \sin \theta_{\zeta}+\zeta_{z}^{2}+\hat{v}_{\theta C}^{2}-\frac{3}{2} \hat{T}_{C}\right) \\
\times \widetilde{\mathcal{F}} d \zeta d \theta_{\zeta} d \zeta_{z},
\end{gathered}
$$

where $\hat{\rho}_{C}, \hat{v}_{\theta C}$, and $\hat{T}_{C}$ in Eq. (17) are, respectively, the (dimensionless) density, flow velocity ( $\theta$ component), and temperature of the cylindrical Couette flow, defined by Eqs. (9a), (9c), and (9e) with $\hat{f}=\hat{f}_{C}\left[\hat{\rho}_{C}\right.$ has appeared in Eq. (13)]; $\hat{f}_{e C}$ is the (dimensionless) local Maxwellian defined by $\hat{f}_{e}$ [Eq. (8)] with $\hat{\rho}=\hat{\rho}_{C}, \hat{v}_{\theta}=\hat{v}_{\theta C}$, and $\hat{T}=\hat{T}_{C}$; (the real parts of $) \epsilon \widetilde{\mathcal{D}}(\hat{t}, \hat{r} ; \alpha) \exp (i \alpha \hat{z}), \epsilon \widetilde{\mathcal{V}_{r}}(\hat{t}, \hat{r} ; \alpha) \exp (i \alpha \hat{z}), \epsilon \widetilde{\mathcal{V}}_{\theta}(\hat{t}, \hat{r}$; $\alpha) \exp (i \alpha \hat{z}), \epsilon \widetilde{\mathcal{V}}_{z}(\hat{t}, \hat{r} ; \alpha) \exp (i \alpha \hat{z})$, and $\epsilon \widetilde{\mathcal{T}}(\hat{t}, \hat{r} ; \alpha) \exp (i \alpha \hat{z})$ are the disturbances corresponding to $\hat{\rho}, \hat{v}_{r}, \hat{v}_{\theta}, \hat{v}_{z}$, and $\hat{T}$, respectively. $\mathcal{L}_{B I}$ and $\mathcal{L}_{B I I}$ in Eqs. (15b) and (15c) are the linear integral operators defined as follows:

$$
\begin{aligned}
\mathcal{L}_{B I}[\tilde{\mathcal{F}}]= & -\frac{2}{\pi} \exp \left(-\zeta^{2}+2 \hat{V}_{I} \zeta \sin \theta_{\zeta}-\zeta_{z}^{2}-\hat{V}_{I}^{2}\right) \\
& \times \int_{-\pi \leqslant \theta_{\zeta}<-\pi / 2, \pi / 2<\theta_{\zeta}<\pi} \zeta^{2} \cos \theta_{\zeta} \widetilde{\mathcal{F}}(\hat{r}=1) d \zeta d \theta_{\zeta} d \zeta_{z},
\end{aligned}
$$

$$
\begin{aligned}
\mathcal{L}_{B I I}[\tilde{\mathcal{F}}]= & \frac{2}{\pi} \exp \left(-\zeta^{2}+2 \hat{V}_{I I} \zeta \sin \theta_{\zeta}-\zeta_{z}^{2}-\hat{V}_{I I}^{2}\right) \\
& \times \int_{\left|\theta_{\zeta}\right|<\pi / 2} \zeta^{2} \cos \theta_{\zeta} \tilde{\mathcal{F}}\left(\hat{r}=L_{I I} / L_{I}\right) d \zeta d \theta_{\zeta} d \zeta_{z} .
\end{aligned}
$$

It is seen from Eqs. (15a)-(15c) that $\widetilde{\mathcal{F}}^{*}\left(\hat{t}, \hat{r}, \zeta, \theta_{\zeta},-\zeta_{z} ; \alpha\right)$, where $\widetilde{\mathcal{F}}^{*}$ is the complex conjugate of $\widetilde{\mathcal{F}}$, satisfies the same equation and boundary conditions as Eqs. (15a)-(15c). Therefore, we can assume without loss of generality that $\widetilde{\mathcal{F}}$ has the following property:

$$
\tilde{\mathcal{F}}\left(\hat{t}, \hat{r}, \zeta, \theta_{\zeta}, \zeta_{z} ; \alpha\right)=\widetilde{\mathcal{F}}^{*}\left(\hat{t}, \hat{r}, \zeta, \theta_{\zeta},-\zeta_{z} ; \alpha\right) .
$$

That is, the real part of $\tilde{\mathcal{F}}$ is even with respect to $\zeta_{z}$, and its imaginary part is odd with respect to $\zeta_{z}$. From Eqs. (21) and (18a)-(18e), it is readily seen that

$$
(\widetilde{\mathcal{D}})_{\mathrm{I}}=\left(\widetilde{\mathcal{V}}_{r}\right)_{\mathrm{I}}=\left(\tilde{\mathcal{V}}_{\theta}\right)_{\mathrm{I}}=\left(\widetilde{\mathcal{V}}_{z}\right)_{\mathrm{R}}=(\widetilde{\mathcal{T}})_{\mathrm{I}} \equiv 0,
$$

where ()$_{R}$ and ()$_{I}$ indicate the real and imaginary parts of the quantity in the parentheses. Therefore, the disturbances 


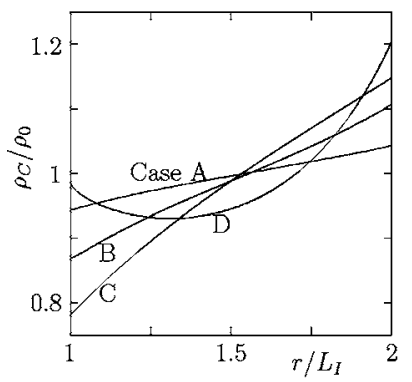

(a)

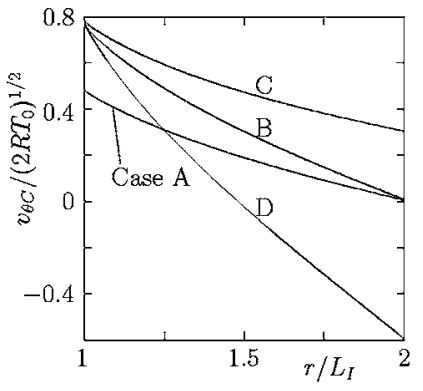

(b)

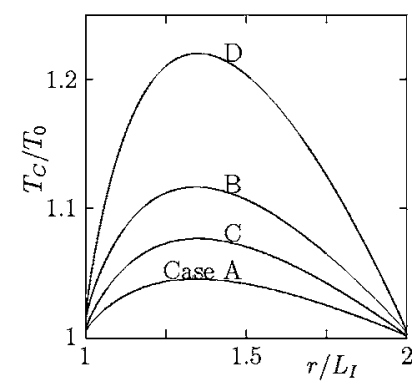

(c)
FIG. 2. Cylindrical Couette flow for relatively small $\mathrm{Kn}$ $\left(L_{I I} / L_{I}=2\right)$. (a) $\rho_{C}$, (b) $v_{\theta C}$, and (c) $T_{C}$. The parameters corresponding to cases $A, B, C$, and $D$ are as follows. Case $A,\left(\mathrm{Kn}, V_{I} I\right.$ $\left.\left(2 R T_{0}\right)^{1 / 2}, V_{I I} /\left(2 R T_{0}\right)^{1 / 2}\right)=(0.02$, $0.5,0)$; case $B,(0.02,0.8,0)$; case $C,(0.0127,0.8,0.3)$; and case $D$, $(0.0127,0.8,-0.6)$. corresponding to $\hat{\rho}, \hat{v}_{r}, \hat{v}_{\theta}, \hat{v}_{z}$, and $\hat{T}$ are reduced to $\epsilon(\tilde{\mathcal{D}})_{\mathrm{R}} \cos \alpha \hat{z}, \quad \epsilon\left(\tilde{\mathcal{V}}_{r}\right)_{\mathrm{R}} \cos \alpha \hat{z}, \quad \epsilon\left(\tilde{\mathcal{V}}_{\theta}\right)_{\mathrm{R}} \cos \alpha \hat{z}, \quad-\epsilon\left(\tilde{\mathcal{V}}_{z}\right)_{\mathrm{I}} \sin \alpha \hat{z}$, $\epsilon(\widetilde{\mathcal{T}})_{\mathrm{R}} \cos \alpha \hat{z}$, respectively.

Now, following the standard procedure in fluid dynamics [18], we assume the time dependence of the disturbance $\tilde{\mathcal{F}}\left(\hat{t}, \hat{r}, \zeta, \theta_{\zeta}, \zeta_{z} ; \alpha\right)$ to be of exponential form, i.e., we let

$$
\tilde{\mathcal{F}}(\hat{t}, \hat{r}, \zeta ; \alpha)=\mathcal{F}(\hat{r}, \zeta ; \alpha, s) \exp (s \hat{t}),
$$

where $s$ is a complex number, and $\zeta$ stands for $\left(\zeta, \theta_{\zeta}, \zeta_{z}\right)$. Substitution of Eq. (23) into Eqs. (15a)-(15c) leads to the following boundary value problem for $\mathcal{F}$ :

$$
\begin{gathered}
\mathcal{L}[\mathcal{F}]=s \mathcal{F} \\
\mathcal{F}=\mathcal{L}_{B I}[\mathcal{F}] \quad \text { at } \hat{r}=1 \text { for }\left|\theta_{\zeta}\right|<\pi / 2 \\
\mathcal{F}=\mathcal{L}_{B I I}[\mathcal{F}] \\
\text { at } \hat{r}=L_{I I} / L_{I} \text { for }-\pi \leqslant \theta_{\zeta}<\pi / 2 \text { and } \pi / 2<\theta_{\zeta}<\pi .
\end{gathered}
$$

This problem forms an eigenvalue problem for eigenvalues $s$ and eigenfunctions $\mathcal{F}$ for a given set of $\mathrm{Kn}, \hat{V}_{I}, \hat{V}_{I I}, L_{I I} / L_{I}$, and $\alpha$. If $s$ and $\mathcal{F}\left(\hat{r}, \zeta, \theta_{\zeta}, \zeta_{z}\right)$ are an eigenvalue and the corresponding eigenfunction, then $s^{*}$ and $\mathcal{F}^{*}\left(\hat{r}, \zeta, \theta_{\zeta},-\zeta_{z}\right)$, where $s^{*}$ and $\mathcal{F}^{*}$ are the complex conjugates of $s$ and $\mathcal{F}$, are also an eigenvalue and the corresponding eigenfunction.

If the eigenfunctions form a complete set in an appropriate function space, the time evolution of the disturbance with an arbitrary initial condition, more specifically, the solution of the initial and boundary value problem formed by Eqs. (15a)-(15c) and an arbitrary initial condition (at $t=0$ ) for $\widetilde{\mathcal{F}}$, can be expressed by the superposition (summation and/or integration) of the form (23) with all the eigenvalues and eigenfunctions of the problem, Eqs. (24a)-(24c). In most practical cases, however, we need to solve numerically eigenvalue problems whose mathematical properties are not clear. If we approximate Eqs. (24a)-(24c) using discretized $\mathcal{F}$ at discrete points in $\hat{r}, \zeta, \theta_{\zeta}$, and $\zeta_{z}$, then we obtain an eigenvalue problem of a large linear algebraic system. By solving such an eigenvalue problem, we obtain a discrete set of eigenvalues, from which we can conjecture the mathematical properties (such as the presence of continuum spectra) of the original eigenvalue problem.

As seen from Eq. (23), the real and imaginary parts of $s$ correspond to the temporal growth rate and the frequency of temporal oscillation of the disturbance, respectively. Therefore, the linear stability of the cylindrical Couette flow against the disturbance with a fixed $\alpha$ can be found by finding the eigenvalue whose real part is maximum among all the eigenvalues. We shall call this value (real part) the maximum growth rate and denote it by $\sigma_{M}$. If $\sigma_{M}$ is less than zero, all the disturbances decay, so that the cylindrical Couette flow is stable. If $\sigma_{M}$ is greater than zero, at least one of the modes of the form (23) grows, so that the cylindrical Couette flow is unstable. If $\sigma_{M}$ is zero, the cylindrical Couette flow is said to be neutrally stable. The point corresponding to the neutrally stable state (neutral stability point) forms hypersurfaces in the $\left(\mathrm{Kn}, \hat{V}_{I}, \hat{V}_{I I}, L_{I I} / L_{I}, \alpha\right)$ space.

\section{B. Numerical analysis}

\section{Cylindrical Couette flow}

We first prepare accurate numerical solutions of the cylindrical Couette flow, the stability of which will be investigated in Sec. III B 2. More specifically, we carry out a numerical analysis using the finite-difference method developed by Sone and Sugimoto in their study of strong evaporation from spherical and cylindrical condensed phases $[37,38]$. A difficulty inherent in the finite-difference analysis is caused by the fact that the molecular velocity distribution function around a convex body generally contains discontinuities $[33,39]$. The method mentioned above is capable of describing the behavior of the discontinuity around a spherical or cylindrical body. The method was adapted to the cylindrical Couette flow setting in Ref. [9], where the bifurcation of flow is shown to occur even under the condition of axially and circumferentially uniform flow when evaporation and condensation take place on the cylinders. Since the numerical method in the present study is essentially the same as that described in detail in Ref. [9], we omit the explanation of the method, giving information about the computational system in Sec. V C.

Some numerical results for the profiles of the density $\hat{\rho}_{C}$ $=\rho_{C} / \rho_{0}$, the $\theta$ component of the flow velocity $\hat{v}_{\theta C}$ $=v_{\theta C} /\left(2 R T_{0}\right)^{1 / 2}$, and the temperature $\hat{T}_{C}=T_{C} / T_{0}$, where $\rho_{C}$, $v_{\theta C}$, and $T_{C}$ are the dimensional quantities corresponding to $\hat{\rho}_{C}, \hat{v}_{\theta C}$, and $\hat{T}_{C}$, are shown in Fig. 2 for relatively small Knudsen numbers in the case of $L_{I I} / L_{I}=2$. The corresponding parameters are shown in the caption. The outer cylinder is at rest in cases $A$ and $B$, rotating in the same direction as the inner in case $C$, and rotating in the opposite direction in case $D$. 


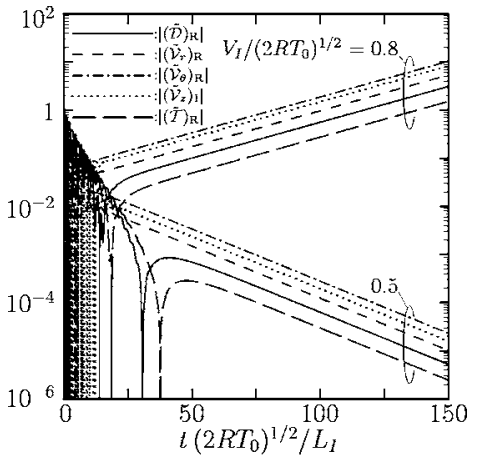

(a)

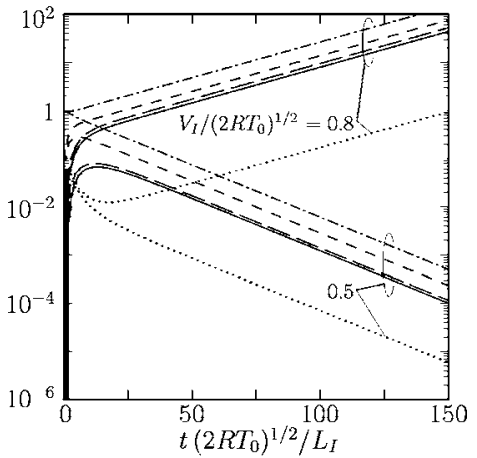

(b)

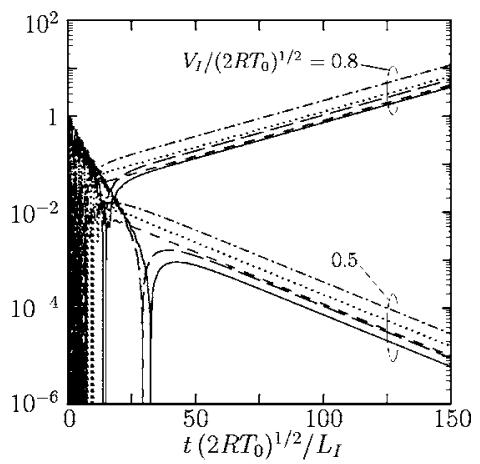

(c)

FIG. 3. Time evolution of the macroscopic variables $\left|(\widetilde{\mathcal{D}})_{\mathrm{R}}\right|$, $\left|\left(\widetilde{\mathcal{V}}_{r}\right)_{\mathrm{R}}\right|,\left|\left(\widetilde{\mathcal{V}}_{\theta}\right)_{\mathrm{R}}\right|,\left|\left(\widetilde{\mathcal{V}}_{z}\right)_{\mathrm{I}}\right|$, and $\left|(\widetilde{\mathcal{T}})_{\mathrm{R}}\right|$ for $\alpha=\pi$ and $V_{I} /\left(2 R T_{0}\right)^{1 / 2}=0.5$ and 0.8 at $\mathrm{Kn}=0.02\left(V_{I I}=0\right.$ and $\left.L_{I I} / L_{I}=2\right)$. (a) $r / L_{I}=1.25$ [initial condition type (a)], (b) $r / L_{I}=1.5$ [initial condition type (b)], (c) $r / L_{I}=1.75$ [initial condition type (c)]. The solid line indicates $\left|(\widetilde{\mathcal{D}})_{\mathrm{R}}\right|$, the short-dashed line $\left|\left(\tilde{\mathcal{V}}_{r}\right)_{\mathrm{R}}\right|$, the dash-dotted line $\left|\left(\tilde{\mathcal{V}}_{\theta}\right)_{\mathrm{R}}\right|$, the dotted line $\left|\left(\widetilde{\mathcal{V}}_{z}\right)_{\mathrm{I}}\right|$, and the long-dashed line $\mid\left(\widetilde{\mathcal{T}}_{\mathrm{R}} \mid\right.$.

\section{Stability of cylindrical Couette flow}

As described in Sec. III A, we can, in principle, solve the eigenvalue problem (24a)-(24c) by reducing it to an eigenvalue problem for a linear algebraic system by means of appropriate discretization. However, the linear operator $\mathcal{L}$ occurring in Eq. (24a) [see Eq. (16)] is rather complicated because it contains many independent variables $\left(\hat{r}, \zeta, \theta_{\zeta}\right.$, and $\zeta_{z}$ ) and integral operators (with respect to $\zeta, \theta_{\zeta}$, and $\zeta_{z}$ ) as well as differential operators (with respect to $\hat{r}$ and $\theta_{\zeta}$ ). Therefore, the discretization of the problem is not a simple task. In the present study, we try to avoid this and calculate the maximum growth rate in the following way. We solve the initial and boundary value problem (15a)-(15c) numerically for an arbitrary initial condition, using the numerical solution $\hat{f}_{C}$. If we take into account the relation (21) and the property described in the second sentence after Eq. (24c), we find that the long-time behavior of $\widetilde{\mathcal{F}}$ is dominated by a function of the form

$$
\mathcal{F}\left(\hat{r}, \zeta, \theta_{\zeta}, \zeta_{z}\right) \exp (s \hat{t})+\mathcal{F}^{*}\left(\hat{r}, \zeta, \theta_{\zeta},-\zeta_{z}\right) \exp \left(s^{*} \hat{t}\right)
$$

with the $s$ whose real part is the largest, provided that $s$ is an isolated eigenvalue. By fitting this form to the long-time behavior of the numerical solution of $\tilde{\mathcal{F}}$, we can in principle determine the maximum growth rate $\sigma_{M}=(s)_{\mathrm{R}}$, the frequency $(s)_{\mathrm{I}}$, and the corresponding eigenfunction $\mathcal{F}$. This process becomes particularly simple when the eigenvalue $s$ giving the maximum growth rate is real, as will be seen below.

The numerical analysis of the initial and boundary value problem (15a)-(15c) is carried out by a finite-difference method similar to that used to obtain the cylindrical Couette flow solution $\hat{f}_{C}$. The difference lies in the fact that the variable $\zeta_{z}$ cannot be eliminated in the problem under consideration and that a time-dependent scheme is employed instead of the time-independent scheme used for the cylindrical Couette flow. We omit the description of the numerical method also here for brevity and give information about the computational system in Sec. V C.

Here, we explain the process of obtaining the maximum growth rate, showing numerical examples. Figure 3 demonstrates the time evolution of the macroscopic quantities obtained from Eqs. (15a)-(15c). To be more specific, the time evolution of the absolute values of the macroscopic variables $(\widetilde{\mathcal{D}})_{\mathrm{R}},\left(\tilde{\mathcal{V}}_{r}\right)_{\mathrm{R}},\left(\tilde{\mathcal{V}}_{\theta}\right)_{\mathrm{R}},\left(\tilde{\mathcal{V}}_{z}\right)_{\mathrm{I}}$, and $(\tilde{\mathcal{T}})_{\mathrm{R}}[$ cf. Eq. $(22)]$ at $r / L_{I}$ $=1.25[(\mathrm{a})], 1.5[(\mathrm{~b})]$, and $1.75[(\mathrm{c})]$ are shown for two different $V_{I}$, i.e., $\hat{V}_{I}=V_{I} /\left(2 R T_{0}\right)^{1 / 2}=0.5$ and 0.8 , in the case where $V_{I I}=0, \mathrm{Kn}=0.02, L_{I I} / L_{I}=2$, and $\alpha=\pi$. The initial conditions for Figs. 3(a)-3(c) are, respectively, the function given by $\tilde{\mathcal{F}}_{e}$ of Eq. (17) with $\tilde{\mathcal{D}}=1$ and $\tilde{\mathcal{V}}_{r}=\tilde{\mathcal{V}}_{\theta}=\tilde{\mathcal{V}}_{z}=\tilde{\mathcal{T}}=0$ [we call this condition type (a)], that with $\tilde{\mathcal{V}}_{\theta}=1$ and $\tilde{\mathcal{D}}=\tilde{\mathcal{V}}_{r}=\tilde{\mathcal{V}}_{z}$ $=\tilde{\mathcal{T}}=0$ [type (b)], and that with $\tilde{\mathcal{T}}=1$ and $\tilde{\mathcal{D}}=\tilde{\mathcal{V}}_{r}=\tilde{\mathcal{V}}_{\theta}=\tilde{\mathcal{V}}_{z}=0$ [type (c)]. In the figures, all the curves for $V_{I} /\left(2 R T_{0}\right)^{1 / 2}$ $=0.5$ tend to approach parallel straight lines after initial time intervals. The same is true for $V_{I} /\left(2 R T_{0}\right)^{1 / 2}=0.8$. Since a logarithmic scale is used for the ordinate, this means that the eigenvalue $s$ giving the maximum growth rate is real and that the time evolution of $(\widetilde{\mathcal{D}})_{\mathrm{R}},\left(\tilde{\mathcal{V}}_{r}\right)_{\mathrm{R}},\left(\tilde{\mathcal{V}}_{\theta}\right)_{\mathrm{R}},\left(\tilde{\mathcal{V}}_{z}\right)_{\mathrm{I}}$, and $(\widetilde{\mathcal{T}})_{\mathrm{R}}$ at each point is dominated by the terms of the following form:

$$
\left[\begin{array}{c}
(\tilde{\mathcal{D}})_{\mathrm{R}} \\
\left(\tilde{\mathcal{V}}_{r}\right)_{\mathrm{R}} \\
\left(\tilde{\mathcal{V}}_{\theta}\right)_{\mathrm{R}} \\
\left(\tilde{\mathcal{V}}_{z}\right)_{\mathrm{I}} \\
(\widetilde{\mathcal{T}})_{\mathrm{R}}
\end{array}\right] \simeq\left[\begin{array}{c}
(\mathcal{D})_{\mathrm{R}} \\
\left(\mathcal{V}_{r}\right)_{\mathrm{R}} \\
\left(\mathcal{V}_{\theta}\right)_{\mathrm{R}} \\
\left(\mathcal{V}_{z}\right)_{\mathrm{I}} \\
(\mathcal{T})_{\mathrm{R}}
\end{array}\right] \exp \left(\sigma_{M} t\right) .
$$

This feature of the time evolution is observed at all the lattice 
points in $\hat{r}$ for the three different initial conditions, types (a), (b), and (c). Therefore, by numerical fitting, we can obtain $(\mathcal{D})_{\mathrm{R}},\left(\mathcal{V}_{r}\right)_{\mathrm{R}},\left(\mathcal{V}_{\theta}\right)_{\mathrm{R}},\left(\mathcal{V}_{z}\right)_{\mathrm{I}}$, and $(\mathcal{T})_{\mathrm{R}}$ as well as $\sigma_{M}$ at all the lattice points in $\hat{r}$ for each initial condition. The numerical values of $\sigma_{M}$ thus obtained show small fluctuations depending on the initial condition, the macroscopic variables, and the lattice points. We take its average over all the macroscopic variables and over all the lattice points for each initial condition and let the average value be the maximum growth rate $\sigma_{M}$. The obtained average value is $\sigma_{M}=-0.4990$ $\times 10^{-1}\left[V_{I} /\left(2 R T_{0}\right)^{1 / 2}=0.5\right]$ and $0.3422 \times 10^{-1}\left[V_{I} /\left(2 R T_{0}\right)^{1 / 2}\right.$ $=0.8]$ for initial condition type (a); $\sigma_{M}=-0.4996$ $\times 10^{-1}\left[V_{I} /\left(2 R T_{0}\right)^{1 / 2}=0.5\right]$ and $0.3414 \times 10^{-1}\left[V_{I} /\left(2 R T_{0}\right)^{1 / 2}\right.$ $=0.8]$ for initial condition type (b); and $\sigma_{M}=-0.4990$ $\times 10^{-1}\left[V_{I} /\left(2 R T_{0}\right)^{1 / 2}=0.5\right]$ and $0.3421 \times 10^{-1}\left[V_{I} /\left(2 R T_{0}\right)^{1 / 2}\right.$ $=0.8]$ for initial condition type (c). The standard deviation from the average value of $\sigma_{M}$ over all the macroscopic variables and lattice points for each initial condition is less than $3.53 \times 10^{-8}$. Therefore, the effect of the initial condition is larger. However, the variation depending on the initial condition seems to be within the error of numerical computation (see Sec. V C). We have performed similar numerical checks for some other values of the parameters and confirmed the above conclusion. Therefore, we adopt the initial condition type (b) to carry out more detailed computation, the results of which will be presented in Sec. V.

On the other hand, the fact that $(\mathcal{D})_{\mathrm{R}},\left(\mathcal{V}_{r}\right)_{\mathrm{R}},\left(\mathcal{V}_{\theta}\right)_{\mathrm{R}},\left(\mathcal{V}_{z}\right)_{\mathrm{I}}$, and $(\mathcal{T})_{\mathrm{R}}$ have been obtained at all the lattice points in $\hat{r}$ means that we have obtained the macroscopic variables corresponding to the eigenfunction $\mathcal{F}(\hat{r}, \zeta ; \alpha, s)$ [Eq. (23)] pertaining to the eigenvalue $s=\sigma_{M}+0 i$. Although the obtained macroscopic variables depend on the initial condition, they should give the same results, independent of the initial condition, once they are normalized. In fact, we have confirmed that the difference of the normalized macroscopic variables depending on the initial condition is within the error of the present numerical computation.

The $\sigma_{M}$ is negative for $V_{I} /\left(2 R T_{0}\right)^{1 / 2}=0.5$ and positive for $V_{I} /\left(2 R T_{0}\right)^{1 / 2}=0.8$. Therefore, $\sigma_{M}$ vanishes at an intermediate value of $V_{I} /\left(2 R T_{0}\right)^{1 / 2}$, which gives the neutral stability point. We obtain $\sigma_{M}$ for various values of $V_{I} /\left(2 R T_{0}\right)^{1 / 2}$ and determine the neutral stability point by interpolation using the natural cubic spline method. The eigenfunction corresponding to the neutral stability point is obtained also by interpolation. We perform this procedure for various values of the set $\left(\mathrm{Kn}, V_{I} /\left(2 R T_{0}\right)^{1 / 2}, V_{I I} /\left(2 R T_{0}\right)^{1 / 2}, L_{I I} / L_{I}, \alpha\right)$. More comprehensive results will be presented in Sec. V.

In the examples shown in Fig. 3, the time evolution after the initial stage does not oscillate, namely, the eigenvalue giving the maximum growth rate is real, as mentioned above. Actually, this is always true near the neutral stability point in the parameter range investigated in the present study. However, in some regions, e.g., $V_{I} /\left(2 R T_{0}\right)^{1 / 2} \gtrsim 1.5$ and $V_{I I} /\left(2 R T_{0}\right)^{1 / 2} \gtrsim 0.5$ for $\alpha=\pi$ in Fig. 12, we have observed decay with oscillation.

\section{DSMC ANALYSIS OF FLOW BETWEEN CYLINDERS}

So far, we have focused on the analysis of small disturbances imposed on the cylindrical Couette flow. On the other hand, some numerical results based on the DSMC method for the flow between rotating cylinders with axial dependence have been reported [24-30]. In this paper, we also carry out some computations that are additional to Refs. $[29,30]$, where the gas molecules are assumed to be elastic hard spheres. These results will be presented in this section. Before that, however, we discuss the comparison between the result based on the BGK model and that based on the DSMC method, taking the much simpler cylindrical Couette flow. Since the DSMC method is explained in many places, we omit its description in the present paper.

For hard-sphere molecules, the Knudsen number $\mathrm{Kn}$ is defined by $\mathrm{Kn}=\ell_{0} / L_{I}$ with $\ell_{0}=\left[\sqrt{2} \pi d^{2}\left(\rho_{0} / m\right)\right]^{-1}$ the mean free path of the gas molecules in the equilibrium state at rest with density $\rho_{0}$, and $m$ and $d$ are, respectively, the mass and diameter of a molecule.

\section{A. Cylindrical Couette flow}

First, we obtain the Couette flow solution of Eqs. (7), (11), and (12) with the right-hand side of Eq. (7) replaced by the Boltzmann collision term for hard-sphere molecules by means of the DSMC method. The resulting profiles of the density $\rho_{C}$, the $\theta$ component of the flow velocity $v_{\theta}$, and the temperature $T_{C}$, in the same case as Fig. 2, are shown in Fig. 4. Recall that $\rho_{0}$ is the average density between the cylinders. In the figure, the BGK result already shown in Fig. 2 is also shown.

It should be mentioned that there is no unique way to compare the results based on two different molecular models, such as the BGK model and hard-sphere molecules. One of the standard ways is to convert the mean free path (or the Knudsen number), assuming that the viscosity is a common quantity for both models. Let $\mu_{0}$ be the viscosity of the gas at density $\rho_{0}$ and temperature $T_{0}$. The relation between $\mu_{0}$ and the corresponding mean free path $\ell_{0}$ is given by $\mu_{0}$ $=(\sqrt{\pi} / 4) \gamma_{1} \rho_{0}\left(2 R T_{0}\right)^{1 / 2} \ell_{0}$ (see Sec. 3.9 of Ref. [33]), where $\gamma_{1}$ is a constant depending on the molecular model: $\gamma_{1}=1$ for the BGK model and 1.270042 for hard-sphere molecules. Therefore, if we assume that $\mu_{0}$ is the common quantity for the BGK model and for hard-sphere molecules, we have the following conversion formula between the Knudsen number for the former $\mathrm{Kn}_{(\mathrm{BGK})}$ and that for the latter $\mathrm{Kn}_{(\mathrm{HS})}$ :

$$
\mathrm{Kn}_{(\mathrm{BGK})}=1.270042 \mathrm{Kn}_{(\mathrm{HS})} \text {. }
$$

In Fig. 4, the DSMC result is plotted after the Knudsen number is converted to that for the BGK model using Eq. (27). In other words, the DSMC computation was carried out for $\mathrm{Kn}_{(\mathrm{HS})}=0.01574751$ (cases $A$ and $B$ ) and 0.01 (cases $C$ and $D$ ), which correspond to $\mathrm{Kn}_{(\mathrm{BGK})}=0.02$ and 0.0127 , respectively. The profile of $v_{\theta}$ for the BGK model shows good agreement with that by the DSMC method (see also Fig. 7 in [15]). The agreement for the profile of $\rho_{C}$ is also relatively good, whereas there is a discrepancy in the profile of $T_{C}$. This is due to the fact that the Prandtl number Pr $(=5 R \mu / 2 \lambda)$ is unity for the BGK model but 0.6607 for hardsphere molecules [see Eqs. (A10a) and (A10b)]. Therefore, if the viscosity is assumed to be common to both molecular 


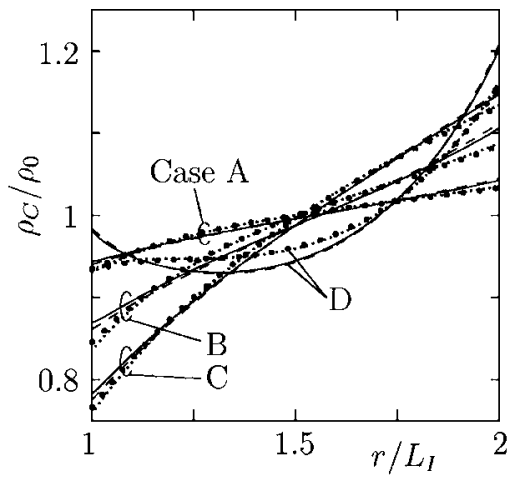

(a)

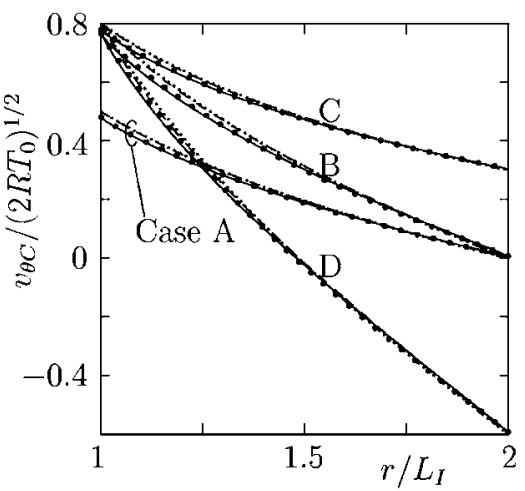

(b)

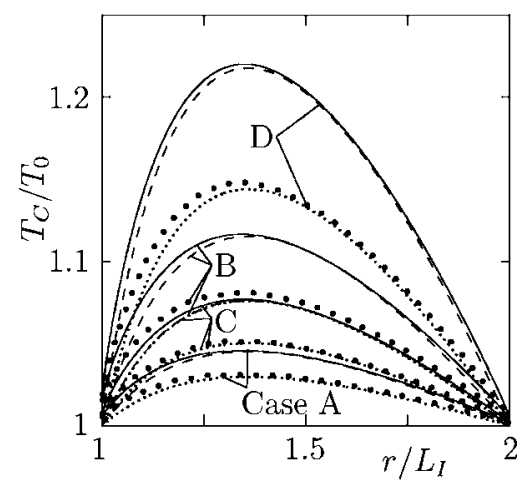

(c)

FIG. 4. DSMC result for the cylindrical Couette flow for relatively small $\mathrm{Kn}$ with some comparison $\left(L_{I I} / L_{I}=2\right.$; see Fig. 2). (a) $\rho_{C}$, (b) $v_{\theta C}$, and (c) $T_{C}$. The parameters corresponding to cases $A, B$, $C$, and $D$ are the same as in Fig. 2, i.e., case $A$, $\left(\mathrm{Kn}, V_{I} /\left(2 R T_{0}\right)^{1 / 2}, V_{I I} /\left(2 R T_{0}\right)^{1 / 2}\right)=(0.02,0.5,0) ; \quad$ case $B, \quad(0.02$, $0.8,0)$; case $C$, $(0.0127,0.8,0.3)$; and case $D,(0.0127,0.8,-0.6)$. Here, the black circle (O) indicates the DSMC result (hard sphere), the solid line the BGK result (cf. Fig. 2), the dashed line the compressible Navier-Stokes result with the BGK viscosity and thermal conductivity, and the dotted line that with hard-sphere viscosity and thermal conductivity.

models, the thermal conductivity becomes rather different. We will use the conversion (27) in Sec. V. It should be noted, however, that this is just a way of comparison and does not guarantee anything.

Incidentally, in Fig. 4 we also show the results for a compressible Navier-Stokes system, which is summarized in the
Appendix, with viscosity and thermal conductivity for the BGK model as well as those for the hard-sphere molecules [the conversion (27) has been used for the hard-sphere case]. More specifically, the system (A11)-(A19) in the case of a steady and axially uniform flow $\left(\partial / \partial \hat{t}=\partial / \partial \hat{z}=0\right.$ and $\hat{v}_{r}$ $\left.=\hat{v}_{z}=0\right)$ is solved numerically with $\hat{\gamma}_{1}=\hat{\gamma}_{2}=\sqrt{\hat{T}}$ for the BGK model and $\hat{\gamma}_{1}=1.270042, \hat{\gamma}_{2}=1.922284$ for hard-sphere molecules. The profiles for the compressible Navier-Stokes system with BGK viscosity and thermal conductivity (or hard-sphere viscosity and thermal conductivity) agree well with those for the BGK model (or for hard-sphere molecules obtained by the DSMC method). This is natural because the Knudsen number considered here is small.

We have also computed the corresponding profiles using the generalized hydrodynamics proposed in Ref. [3]. The equations are of a compressible Navier-Stokes type, but their viscosity and thermal conductivity are more involved. Although the Lennard-Jones fluid is considered in Ref. [3], the application to other molecular models, such as the BGK model and hard-sphere molecules, is obvious [40]. For the sake of comparison, we adopted these two molecular models rather than the Lennard-Jones potential. The aim of the generalized hydrodynamics is to extend the applicability of the macroscopic approach to larger Knudsen numbers, and it reduces to the compressible Navier-Stokes system when the Knudsen number is small. In fact, in the case of Fig. 4, the results are indistinguishable from the corresponding results for the compressible Navier-Stokes system.

In the DSMC computation, the following computational system is used. The domain $L_{I} \leqslant r \leqslant L_{I I}$ is divided into 400 uniform cells; the total number of simulation particles is $4 \times 10^{4}$; and the time step $\Delta t$ is $\Delta t=5 \times 10^{-4} L_{I}\left(2 R T_{0}\right)^{-1 / 2}$ $=5 \times 10^{-4}(2 / \sqrt{\pi}) t_{0} \mathrm{Kn}^{-1}$, where $t_{0}$ is the mean free time corresponding to $\ell_{0}$. The initial condition is the equilibrium state at rest at density $\rho_{0}$ and temperature $T_{0}$.

\section{B. Taylor-vortex flow}

Now we consider the flow between the cylinders without axial uniformity. Since the setting for the DSMC computation in this case is slightly different from that given in Sec. II, we first describe the difference.

Instead of the infinite domain in the $z$ direction, we consider a gas in an annular domain $L_{I} \leqslant r \leqslant L_{I I}, 0 \leqslant z \leqslant L_{z}$ bounded by the two cylinders and top and bottom boundaries $\left(z=L_{z}\right.$ and $\left.z=0\right)$. We assume that the gas molecules are reflected specularly on the latter boundaries, i.e.,

$$
f\left(t, r, z, \xi_{r}, \xi_{\theta}, \xi_{z}\right)=f\left(t, r, z, \xi_{r}, \xi_{\theta},-\xi_{z}\right),
$$

at $z=0$ and $L_{z}$. In addition, the BGK collision term in Eq. (1) is replaced by the Boltzmann collision term for hard-sphere molecules. Then, the Boltzmann equation with the boundary conditions (4a), (4b), (5a), (5b), and (28) is solved by the DSMC method for a given initial condition at $t=0$,

$$
f(0, r, z, \boldsymbol{\xi})=f_{0}(r, z, \boldsymbol{\xi}) .
$$

In this setting, the reference density $\rho_{0}$ is the average density of the gas over the annular domain. 


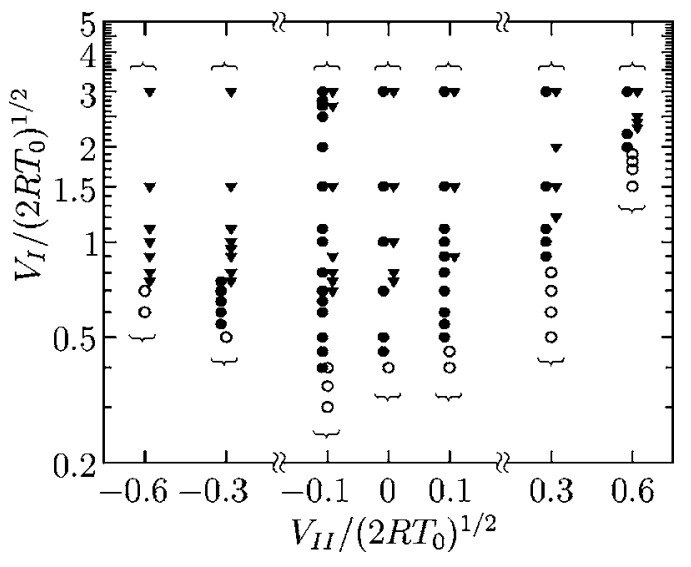

FIG. 5. DSMC result for the existence range of the steady solutions in the $\left(V_{I I} /\left(2 R T_{0}\right)^{1 / 2}, V_{I} /\left(2 R T_{0}\right)^{1 / 2}\right)$ plane at $\mathrm{Kn}=0.01$ $\left(L_{I I} / L_{I}=2\right.$ and $\left.L_{z} / L_{I}=1\right)$. The symbols $\bigcirc, \boldsymbol{O}$, and $\boldsymbol{\nabla}$ indicate that a cylindrical Couette flow, a single-vortex flow, and a double-vortex flow, respectively, are obtained at the corresponding points. Here, the symbols stand for the data precisely at $V_{I I} /\left(2 R T_{0}\right)^{1 / 2}=-0.6$, $-0.3,-0.1,0,0.1,0.3$, and 0.6 , but $\bigcirc$ 's ( $\boldsymbol{\nabla}$ 's) are shifted leftward (rightward) slightly for legibility.

If we carry out the above DSMC computation, we find some different types of final steady solution. Figure 5, which is taken from Ref. [30], shows the types of final steady solution when $L_{I I} / L_{I}=2, L_{z} / L_{I}=1$, and $\mathrm{Kn}=0.01$. To be more specific, the computation for various values of $V_{I} /\left(2 R T_{0}\right)^{1 / 2}$ and $V_{I I} /\left(2 R T_{0}\right)^{1 / 2}$ gives three different types of steady flow pattern: an axially uniform flow (cylindrical Couette flow); a Taylor-vortex flow with a single vortex (single-vortex flow); and a Taylor-vortex flow with double vortices arranged in the axial direction (double-vortex flow). Figure 5 is the map of the flow patterns in the $\left(V_{I I} /\left(2 R T_{0}\right)^{1 / 2}, V_{I} /\left(2 R T_{0}\right)^{1 / 2}\right)$ plane. A cylindrical Couette flow is obtained at the points with $\bigcirc$, a single-vortex flow at the points with $\bullet$, and a double-vortex flow at the points with $\boldsymbol{\nabla}$. The symbols indicate the results at $V_{I I} /\left(2 R T_{0}\right)^{1 / 2}=-0.6,-0.3,-0.1,0,0.1,0.3$, and 0.6 , but $\bullet$ 's ( $\boldsymbol{\nabla}$ 's) are shifted leftward (rightward) slightly for legibility. A negative $V_{I I}$ means that the outer cylinder is rotating in the direction opposite to the inner. In this computation, the initial condition (29) is chosen in such a way that we can extend the range of each type of solution as wide as possible (see Ref. [30] for the details). There is a significant overlap between the region of a single-vortex flow and that of a double-vortex flow when $V_{I I} /\left(2 R T_{0}\right)^{1 / 2} \geqslant-0.1$. For $V_{I I} /\left(2 R T_{0}\right)^{1 / 2}=-0.3$, a single-vortex flow is limited to a rather narrow range of intermediate $V_{I} /\left(2 R T_{0}\right)^{1 / 2}$, and its overlap with the range of a double-vortex flow is small. There is no region of a singlevortex flow for $V_{I I} /\left(2 R T_{0}\right)^{1 / 2}=-0.6$. In Fig. 6, flow patterns of a single- and a double-vortex flow are shown for the point $\left(V_{I I} /\left(2 R T_{0}\right)^{1 / 2}, V_{I} /\left(2 R T_{0}\right)^{1 / 2}\right)=(0,1.5)$ in Fig. 5 where the two types of flow coexist. The panel (a) corresponds to the single-vortex flow, and the panel (b) to the double-vortex flow. The arrow indicates the flow velocity vector $\left(v_{r}, v_{z}\right)$ in the $(r, z)$ plane at its starting point, and the scale of $\left(v_{r}^{2}\right.$ $\left.+v_{z}^{2}\right)^{1 / 2} /\left(2 R T_{0}\right)^{1 / 2}=0.2$ is shown in each figure. The contour line $v_{\theta} /\left(2 R T_{0}\right)^{1 / 2}=$ const is also shown in the figure. These results, as well as some additional results obtained in the

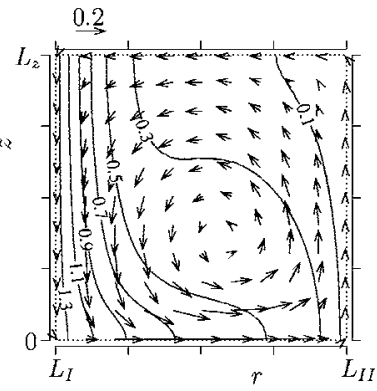

(a)

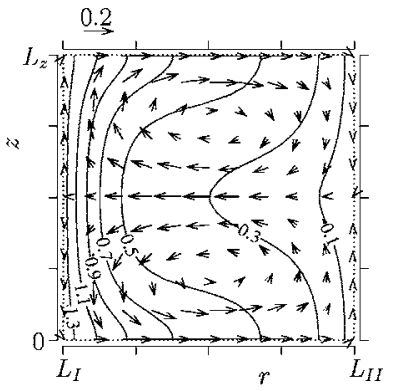

(b)
FIG. 6. DSMC result for the flow field of single- and doublevortex flows for $V_{I} /\left(2 R T_{0}\right)^{1 / 2}=1.5$ and $V_{I I} /\left(2 R T_{0}\right)^{1 / 2}=0$ at $\mathrm{Kn}$ $=0.01 \quad\left(L_{I I} / L_{I}=2\right.$ and $\left.L_{z} / L_{I}=1\right)$. (a) Single-vortex flow and (b) double-vortex flow. The arrow indicates the flow velocity $\left(v_{r}, v_{z}\right)$ in the $(r, z)$ plane at its starting point, and the scale of $\left(v_{r}^{2}\right.$ $\left.+v_{z}^{2}\right)^{1 / 2} /\left(2 R T_{0}\right)^{1 / 2}=0.2$ is shown in each figure. The contour lines $v_{\theta} /\left(2 R T_{0}\right)^{1 / 2}=$ const are also shown in the figure.

present study, will be compared with the result of the linearstability analysis in the next section.

In Figs. 5 and 6, the original Knudsen number for hardsphere molecules is used. Instead, the DSMC results in Fig. 10 and in Fig. 12 appearing in Sec. V are plotted after the Knudsen number is converted to $\mathrm{Kn}_{(\mathrm{BGK})}$ by the use of Eq. (27).

The computational system used in the present study as well as in Ref. [30] is as follows. The square cross section $\left(L_{I} \leqslant r \leqslant L_{I I}, 0 \leqslant z \leqslant L_{z}\right.$ with $L_{I I} / L_{I}=2$ and $\left.L_{z} / L_{I}=1\right)$ is divided into $120 \times 120$ square cells of a uniform size; the total number of simulation particles is $144 \times 10^{4}$; and the time step $\Delta t$ is $\Delta t=5 \times 10^{-4} L_{I}\left(2 R T_{0}\right)^{-1 / 2}=5 \times 10^{-4}(2 / \sqrt{\pi}) t_{0} \mathrm{Kn}^{-1}$, where $t_{0}$ is the mean free time corresponding to $\ell_{0}$.

\section{RESULTS AND DISCUSSION}

In this section, we will present some results of the stability analysis described in Sec. III B 2. We first investigate the effect of gas rarefaction in the case where the outer cylinder is at rest and then discuss the effect of rotation of the outer cylinder. Throughout this section, the ratio of the cylinder radii is fixed to be $L_{I I} / L_{I}=2$.

\section{A. Effect of gas rarefaction}

Let us consider the case where the outer cylinder is at rest and investigate the effect of the Knudsen number as well as that of the rotation speed of the inner cylinder on the stability of the cylindrical Couette flow.

Figure 7 shows the maximum growth rate $\sigma_{M}$ versus the dimensionless surface velocity $\hat{V}_{I}=V_{I} /\left(2 R T_{0}\right)^{1 / 2}$ of the inner cylinder for different Knudsen numbers. The data for $\alpha=\pi$, $2 \pi$, and $3 \pi$ are indicated by the symbol $\bullet$, and the data for each $\alpha$ are connected by the natural cubic spline curve (the solid curve). The curves for larger $\alpha$ are located downward. This means that the disturbance with a higher frequency (in the $z$ direction) has a lower growth rate. In Fig. 7, $\sigma_{M}$ for each $\alpha$ tends to have a maximum value at a certain $V_{I} /\left(2 R T_{0}\right)^{1 / 2}$. As the Knudsen number increases, the maxi- 


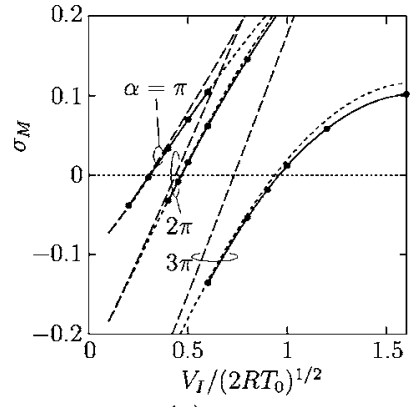

(a) $\mathrm{Kn}=0.01$

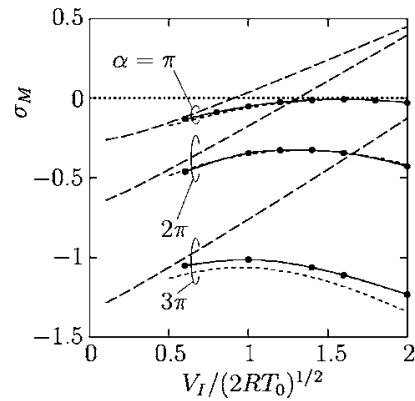

(c) $\mathrm{Kn}=0.03$

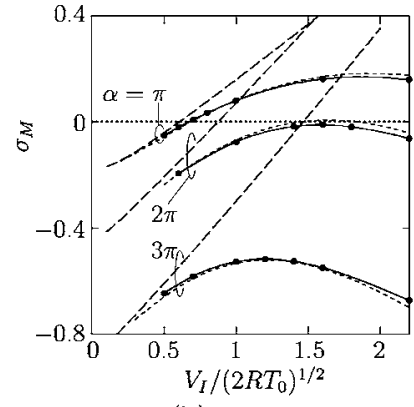

(b) $\mathrm{Kn}=0.02$

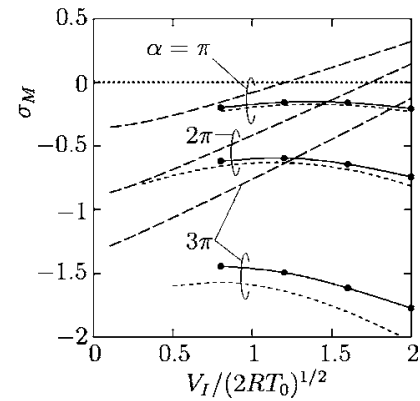

(d) $\mathrm{Kn}=0.04$

FIG. 7. Maximum growth rate $\sigma_{M}$ versus $V_{I} /\left(2 R T_{0}\right)^{1 / 2}$ for $\alpha$ $=\pi, 2 \pi$, and $3 \pi\left(V_{I I}=0\right.$ and $\left.L_{I I} / L_{I}=2\right) . \mathrm{Kn}=$ (a) 0.01 , (b) 0.02 , (c) 0.03 , and (d) 0.04 . The result based on the BGK equation is shown by - and the solid line is the cubic spline curve connecting these points; the corresponding results based on the INS and CNS systems are also shown by the long- and short-dashed lines, respectively.

mum values decrease: in Fig. 7(b) the maximum values for $\alpha=2 \pi$ and $3 \pi$ are negative; and in Figs. 7(c) and 7(d) the maximum values for all three $\alpha$ 's are negative. Therefore, there is no unstable region for these disturbances when the Knudsen number is larger than a certain value.

For the sake of comparison in this and the following subsections, we have also carried out corresponding stability analyses on the basis of continuum systems, a system of the incompressible Navier-Stokes (INS) type and one of the compressible Navier-Stokes (CNS) type, with the viscosity and thermal conductivity corresponding to the BGK model. The systems are summarized in the Appendix. To be more precise, the INS system is composed of Eqs. (A2)-(A8) with $\gamma_{1}=1$, and the CNS system Eqs. (A11)-(A19) with $\hat{\gamma}_{1}=\hat{\gamma}_{2}$ $=\sqrt{\hat{T}}$. Since the case of high speed rotation of the cylinders is considered in the present study, the INS system is not expected to give reasonable results. In Fig. 7, the result based on the INS system is shown by the long-dashed line, while that based on the CNS system is shown by the short-dashed line (only the cubic spline curves connecting the numerical data are shown for legibility). The CNS results are relatively close to the BGK results and thus show the same tendency. Instead, the INS results show different behavior, that is, $\sigma_{M}$ increases monotonically with the increase of $V_{I} /\left(2 R T_{0}\right)^{1 / 2}$.

Figure 8 shows the neutral stability curve (i.e., the locus of the neutral stability point) in the $\left(\mathrm{Kn}, V_{I} /\left(2 R T_{0}\right)^{1 / 2}\right)$ plane for $\alpha=\pi$. More precisely, the symbol $\boldsymbol{\Delta}$ indicates the numerical data for the neutral stability points, which are also

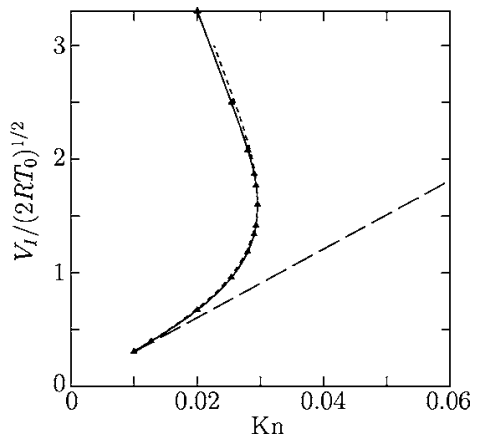

FIG. 8. Neutral stability curve in the $\left(\mathrm{Kn}, V_{I} /\left(2 R T_{0}\right)^{1 / 2}\right)$ plane for $\alpha=\pi\left(V_{I I}=0\right.$ and $\left.L_{I I} / L_{I}=2\right)$. The neutral stability points based on the BGK equation are shown by $\boldsymbol{\Delta}$, and the solid line is the cubic spline curve connecting these points. The corresponding neutral stability curves based on the INS and CNS systems are also shown by the long- and short-dashed lines, respectively.

contained in Table I [the data with the entry $V_{I I} /\left(2 R T_{0}\right)^{1 / 2}$ $=0]$, and the solid curve the natural cubic spline curve (in parametric representation) connecting the data. The cylindrical Couette flow is unstable in the left-hand side of the curve. The results based on the INS and CNS systems are also shown by the long- and short-dashed lines, respectively (only the curves connecting the numerical data are shown). The curve for the BGK system and that for the CNS system are close to each other, and the cylindrical Couette flow based on these two systems is stabilized more rapidly than that based on the INS system as the Knudsen number increases. According to the BGK result, the cylindrical Couette flow is stable for the disturbance with $\alpha=\pi$ for any value of $V_{I} /\left(2 R T_{0}\right)^{1 / 2}$ when the Knudsen number is larger than 0.0296. In the left-hand figures of Figs. 9(a)-9(c), we show the profiles of the macroscopic quantities $(\mathcal{D})_{\mathrm{R}},\left(\mathcal{V}_{r}\right)_{\mathrm{R}},\left(\mathcal{V}_{\theta}\right)_{\mathrm{R}}$, $\left(\mathcal{V}_{z}\right)_{\mathrm{I}}$, and $(\mathcal{T})_{\mathrm{R}}$ pertaining to the eigenfunction $\mathcal{F}$ at three points on the neutral curve (the solid curve) in Fig. 8, i.e., $\left(\mathrm{Kn}, V_{I} /\left(2 R T_{0}\right)^{1 / 2}\right)=(0.01,0.308) \quad[(\mathrm{a})],(0.02,0.672) \quad[(\mathrm{b})]$, and $(0.029,1.337)[(\mathrm{c})]$. In the figures, the quantities are nor-

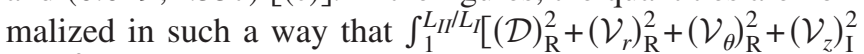
$\left.+(\mathcal{T})_{\mathrm{R}}^{2}\right] \hat{r} d \hat{r}=1$. In the right-hand figures of Figs. 9(a)-9(c), we show the corresponding flow velocity vector of the disturbance $\left(\left(\mathcal{V}_{r}\right)_{\mathrm{R}} \cos \alpha \hat{z},-\left(\mathcal{V}_{z}\right)_{\mathrm{I}} \sin \alpha \hat{z}\right)$ in the $\left(r / L_{I}, z / L_{I}\right)$ plane. The scale based on the above normalization is shown in the figures. At these neutral points, the disturbance is of single-vortex type in the square domain $L_{I} \leqslant r \leqslant L_{I I}=2 L_{I}$ and $0 \leqslant z \leqslant L_{z}=L_{I}$. The features shown in Fig. 9 do not change much in the regions near the corresponding neutral points.

In Fig. 10, the neutral stability curve for the BGK system for $\alpha=\pi$, which has already been presented in Fig. 8, is shown together with the result of the direct numerical analysis of the flow between the cylinders by the DSMC method (see Sec. IV). Since the DSMC computation deals with a finite domain in the $z$ direction $\left(0 \leqslant z \leqslant L_{z}=L_{I}\right)$ bounded by specularly reflecting boundaries at $z=0$ and $L_{I}$, the corresponding value of $\alpha$ is restricted to $\pi, 2 \pi, 3 \pi, \ldots$. Figure 10 also contains the corresponding neutral stability curve based on the CNS system for hard-sphere molecules, i.e., Eqs. (A11)-(A19) with $\hat{\gamma}_{1}=1.270042$ and $\hat{\gamma}_{2}=1.922284$. Both of 
TABLE I. Neutral stability points for $\alpha=\pi$ in the case of $L_{I I} / L_{I}=2$.

\begin{tabular}{cccccc}
\hline \hline \multicolumn{2}{c}{$V_{I I} /\left(2 R T_{0}\right)^{1 / 2}=0$} & \multicolumn{2}{c}{$\mathrm{Kn}=0.0127$} & \multicolumn{2}{c}{$\mathrm{Kn}=0.0254$} \\
\hline $\mathrm{Kn}$ & $V_{I} /\left(2 R T_{0}\right)^{1 / 2}$ & $V_{I I} /\left(2 R T_{0}\right)^{1 / 2}$ & $V_{I} /\left(2 R T_{0}\right)^{1 / 2}$ & $V_{I I} /\left(2 R T_{0}\right)^{1 / 2}$ & $V_{I} /\left(2 R T_{0}\right)^{1 / 2}$ \\
\hline 0.01 & 0.308 & -0.8 & 0.901 & -0.8 & 1.756 \\
0.0127 & 0.397 & -0.6 & 0.741 & -0.6 & 1.324 \\
0.02 & 0.672 & -0.4 & 0.580 & -0.4 & 1.057 \\
0.0254 & 0.958 & -0.2 & 0.433 & -0.2 & 0.912 \\
0.028 & 1.184 & 0 & 0.397 & -0.1 & 0.903 \\
0.029 & 1.337 & 0.1 & 0.484 & 0 & 0.958 \\
0.0293 & 1.417 & 0.2 & 0.649 & 0.1 & 1.119 \\
0.0296 & 1.6 & 0.3 & 0.903 & 0.14 & 1.246 \\
0.0293 & 1.770 & 0.35 & 1.089 & 0.167 & 1.4 \\
0.029 & 1.866 & 0.4 & 1.379 & 0.178 & 1.6 \\
0.028 & 2.086 & 0.423 & 1.7 & 0.168 & 1.8 \\
0.0254 & 2.499 & 0.423 & 2 & 0.14 & 2.005 \\
0.02 & 3.306 & 0.4 & 2.417 & 0.1 & 2.191 \\
& & 0.35 & 2.883 & 0 & 2.499 \\
\hline \hline
\end{tabular}

the DSMC and CNS results (for hard-sphere molecules) in the figure are shown after the Knudsen number is converted to that of the BGK model by the use of Eq. (27). The symbols $\bigcirc, \odot$, and $\diamond$ are the DSMC results taken from Ref. [29], where the initial condition is the equilibrium state at rest with temperature $T_{0}$ and density $\rho_{0}$, that is, the inner cylinder starts to rotate impulsively at the initial time. The symbols $\square$ and $\square$ indicate the results obtained in the present study, using the cylindrical Couette flow obtained by imposing the axial uniformity as the initial condition. The latter initial condition introduces much less disturbances than the former. The symbols $\bigcirc$ and $\square$ indicate that the final steady solution is the cylindrical Couette flow, and $\boldsymbol{O}$ and $\mathbf{\square}$ indicate that a single-vortex flow is formed in the $(r, z)$ plane in the final steady state. The symbol $\diamond$ indicates that it is difficult to determine the type of the solution with the DSMC computation.

The neutral stability curve for the BGK model is close to that for the CNS system with hard-sphere viscosity and thermal conductivity when $\mathrm{Kn} \lesssim 0.02$. However, they differ significantly for larger $\mathrm{Kn}$. Since the former is very close to the CNS result for the BGK model (Fig. 8), we can expect that the CNS result for hard-sphere molecules in Fig. 10 is close to the neutral stability curve based on the Boltzmann equation for hard-sphere molecules. The above discrepancy just means that the conversion (27) is not an appropriate way to compare the result for the BGK model and that for hardsphere molecules when the speed of rotation of the inner cylinder is high (note that the Mach number of the surface speed of the inner cylinder reaches unity at the neutral stability point when $\mathrm{Kn}=0.0254$ ). The reason can be seen from the comparison between the CNS system for the BGK model and that for hard-sphere molecules. When the rotation speed of the inner cylinder is very high, the temperature of the gas $T$ becomes significantly higher than the temperature of the cylinder $T_{0}$, i.e., $\hat{T}$ becomes larger than unity. As seen from
Eq. (A10a), the viscosity in the CNS system for the BGK model becomes larger than that in the CNS system for hardsphere molecules. Therefore, though the viscosity is set to be common at $T_{0}$ by Eq. (27), the effective viscosity is larger in the CNS system for the BGK model. This means that the cylindrical Couette flow for the BGK model is more stabilized. In spite of this drawback, we will still use the conversion (27) in Sec. V B, since we make a comparison at Kn $=0.0127$. The neutral stability curve for the CNS system with hard-sphere viscosity and thermal conductivity seems to be consistent with the DSMC result.

\section{B. Effect of rotation of the outer cylinder}

Now let us investigate the effect of rotation of the outer cylinder.

In Fig. 11, the neutral stability curves, based on the BGK system, for $\alpha=\pi$ at two Knudsen numbers $\mathrm{Kn}=0.0127$ and 0.0254 , are shown in the $\left(V_{I I} /\left(2 R T_{0}\right)^{1 / 2}, V_{I} /\left(2 R T_{0}\right)^{1 / 2}\right)$ plane. To be more precise, the symbol $\boldsymbol{\Delta}$ indicates the numerical results for the neutral stability points, which are also contained in Table I (the data with the entries $\mathrm{Kn}=0.0127$ and $\mathrm{Kn}=0.0254$ ), and the solid curve is the natural cubic spline curve connecting the data. The cylindrical Couette flow is unstable in the upper side of the curves. The corresponding curves based on the INS and CNS equations (with the BGK viscosity and thermal conductivity) are also shown by the long-dashed and short-dashed lines, respectively (only the curves connecting the numerical data are shown in the figure). We recall that the negative value of $V_{I I} /\left(2 R T_{0}\right)^{1 / 2}$ means that the direction of rotation of the outer cylinder is opposite to the inner.

For $\mathrm{Kn}=0.0127$, the curve for the BGK system and that for the CNS system show good agreement in a wide range of $V_{I I} /\left(2 R T_{0}\right)^{1 / 2}$, but the difference is not negligible for $V_{I I} /\left(2 R T_{0}\right)^{1 / 2}$ larger than 0.3 . For example, at $V_{I I} /\left(2 R T_{0}\right)^{1 / 2}$ 

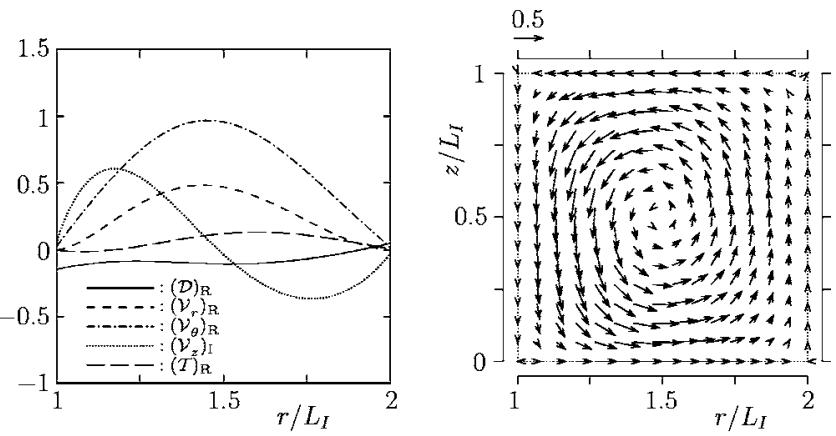

(a)
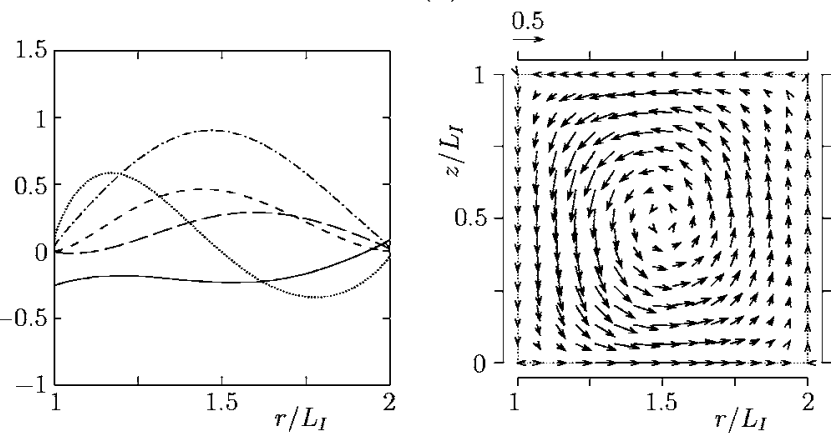

(b)
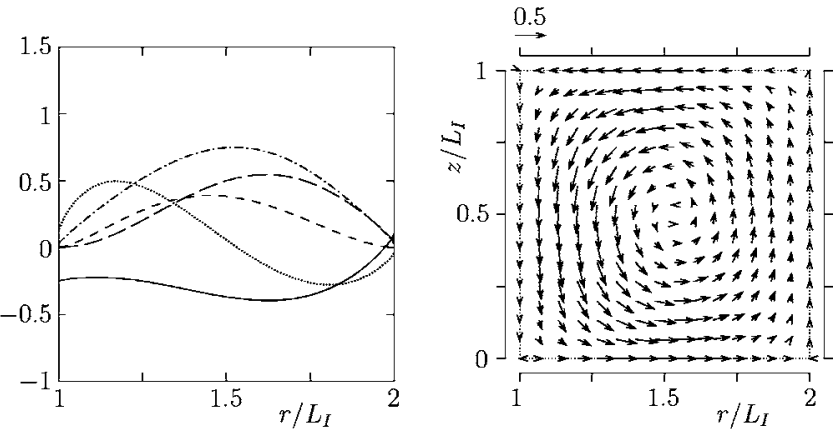

(c)

FIG. 9. Macroscopic quantities pertaining to $\mathcal{F}$ at three neutral stability points for $\alpha=\pi\left(V_{I I}=0\right.$ and $\left.L_{I I} / L_{I}=2\right)$. $\left(\mathrm{Kn}, V_{I} /\left(2 R T_{0}\right)^{1 / 2}\right)$ $=$ (a) $(0.01,0.308)$, (b) $(0.02,0.672)$, and (c) $(0.029,1.337)$. In the left-hand figures, the solid line indicates $(\mathcal{D})_{\mathrm{R}}$, short-dashed line $\left(\mathcal{V}_{r}\right)_{\mathrm{R}}$, dash-dotted line $\left(\mathcal{V}_{\theta}\right)_{\mathrm{R}}$, dotted line $\left(\mathcal{V}_{z}\right)_{\mathrm{I}}$, and long-dashed line $(\mathcal{T})_{\mathrm{R}}$. These quantities are normalized in such a way that $\int_{1}^{L_{I I} / L_{I}}\left[(\mathcal{D})_{\mathrm{R}}^{2}+\left(\mathcal{V}_{r}\right)_{\mathrm{R}}^{2}+\left(\mathcal{V}_{\theta}\right)_{\mathrm{R}}^{2}+\left(\mathcal{V}_{z}\right)_{\mathrm{I}}^{2}+(\mathcal{T})_{\mathrm{R}}^{2}\right] \hat{r} d \hat{r}=1$. In the right-hand figures, the arrows show the flow velocity vectors of the disturbance $\left(\left(\mathcal{V}_{r}\right)_{\mathrm{R}} \cos \alpha \hat{z},-\left(\mathcal{V}_{z}\right)_{\mathrm{I}} \sin \alpha \hat{z}\right)$ at their starting points in the $\left(r / L_{I}, z / L_{I}\right)$ plane. The scale based on the above normalization is shown in the figures.

$=0.45$, the BGK result shows that the cylindrical Couette flow is stable for any value of $V_{I} /\left(2 R T_{0}\right)^{1 / 2}$, whereas the CNS result shows that it is unstable in the range 1.69 $\leqslant V_{I} /\left(2 R T_{0}\right)^{1 / 2} \leqslant 2.18$. For $\mathrm{Kn}=0.0254$, the difference between the $\mathrm{BGK}$ and $\mathrm{CNS}$ results is not negligible in a wider range of $V_{I I} /\left(2 R T_{0}\right)^{1 / 2}$. Because of high speed of rotation of the cylinders, the results for the INS equation naturally show large deviation from those for the CNS and thus BGK equations. The three curves agree well in the vicinity of $V_{I I} /\left(2 R T_{0}\right)^{1 / 2}=0$ when $\mathrm{Kn}=0.0127$ because $V_{I} /\left(2 R T_{0}\right)^{1 / 2}$ there is relatively small.

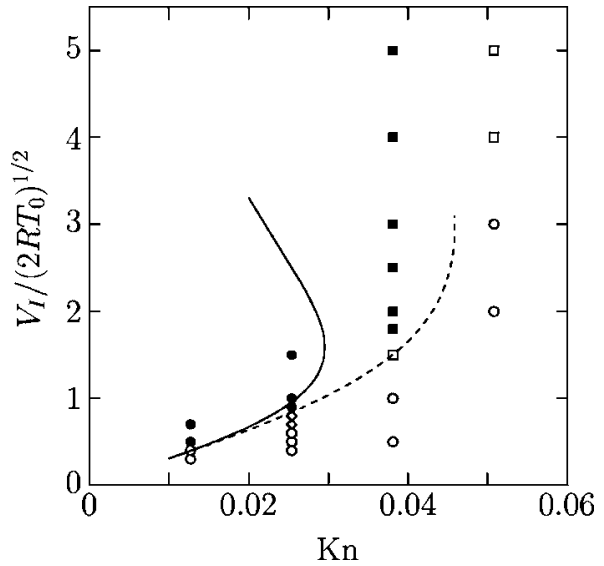

FIG. 10. Comparison of the neutral stability curve for $\alpha=\pi$ $\left(V_{I I}=0\right.$ and $\left.L_{I I} / L_{I}=2\right)$ with the DSMC results in the $\left(\mathrm{Kn}, V_{I} /\left(2 R T_{0}\right)^{1 / 2}\right)$ plane. The solid line is the neutral stability curve (spline curve) based on the BGK equation shown in Fig. 8, and the dashed line that based on the CNS system with hard-sphere viscosity and thermal conductivity. The symbols are the DSMC results with specularly reflecting boundaries at $z=0$ and $L_{I}$. The circles $\bigcirc$ and $\bullet$ and the diamonds $\diamond$ are the results from Ref. [29], where the equilibrium state at rest is taken as the initial condition; the squares $\square$ and $\boldsymbol{\square}$ are the results obtained by using the cylindrical Couette flow as the initial condition. The long-time solution is the cylindrical Couette flow at the points with the symbols $\bigcirc$ and $\square$, whereas it is the Taylor-vortex flow with a single vortex at the points with the symbols $\boldsymbol{Q}$ and $\mathbf{\square}$. The diamond $\diamond$ indicates that, at these points, it is difficult to determine the type of solution.

In Fig. 12, the neutral stability curves, based on the BGK system, at $\mathrm{Kn}=0.0127$ for $\alpha=\pi$ and $2 \pi$ are shown together with some DSMC results (hard-sphere molecules) [30] taken from Fig. 5 for $\mathrm{Kn}=0.01$ (recall that the setting for the DSMC computation corresponds to $\alpha=\pi, 2 \pi, 3 \pi, \ldots)$. The correspondence of the Knudsen numbers is based on the conversion formula (27). The meaning of the symbols is the same as Fig. 5. That is, a cylindrical Couette flow is obtained at the points with $\bigcirc$, a single-vortex flow at the points with

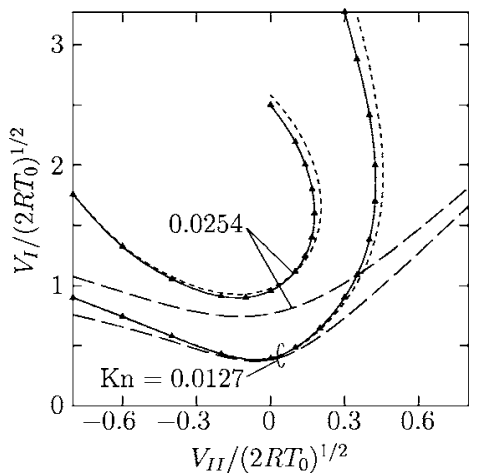

FIG. 11. Neutral stability curves in the $\left(V_{I I} /\right.$ $\left.\left(2 R T_{0}\right)^{1 / 2}, V_{I} /\left(2 R T_{0}\right)^{1 / 2}\right)$ plane for $\alpha=\pi\left(L_{I I} / L_{I}=2\right)$. The neutral stability points for $\mathrm{Kn}=0.0127$ and 0.0254 based on the BGK equation are shown by $\boldsymbol{\Lambda}$, and the solid curves are the cubic spline curves connecting the data. The corresponding curves based on the INS and CNS systems are also shown by the long- and short-dashed lines, respectively. 


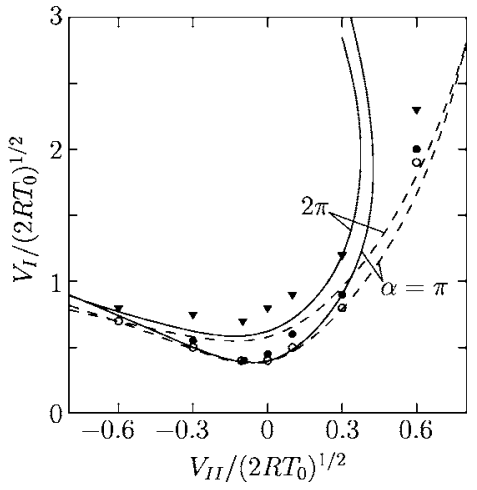

FIG. 12. Comparison of the neutral stability curves for $\alpha=\pi$ and $2 \pi$ at $\mathrm{Kn}=0.0127\left(L_{I I} / L_{I}=2\right)$ with the DSMC results in the $\left(V_{I I} /\left(2 R T_{0}\right)^{1 / 2}, V_{I} /\left(2 R T_{0}\right)^{1 / 2}\right)$ plane. The solid lines indicate the neutral stability curves (cubic spline curves) based on the BGK equation (the curve for $\alpha=\pi$ is shown in Fig. 11), and the dashed lines those based on the CNS system with hard-sphere viscosity and thermal conductivity. The symbols are the DSMC results from Fig. 5 with the specularly reflecting boundaries at $z=0$ and $L_{I}$. The white circles $\bigcirc$ indicate the largest values of $V_{I} /\left(2 R T_{0}\right)^{1 / 2}$ where the cylindrical Couette flow is obtained, the black circles the smallest values where the Taylor-vortex flow with a single vortex is obtained, and the black triangles $\boldsymbol{\nabla}$ the smallest values where the Taylor-vortex flow with double vortices, arranged in the $z$ direction, is obtained.

, and a double-vortex flow at the points with $\nabla$. More precisely, for each $V_{I} /\left(2 R T_{0}\right)^{1 / 2}$, the highest $\bigcirc$ and the lowest and $\boldsymbol{\nabla}$ are picked up from Fig. 5 and plotted in Fig. 12. This figure also contains the corresponding neutral stability curves based on the CNS system with hard-sphere viscosity and thermal conductivity, which are indicated by the dashed lines.

In the left-hand figures of Figs. 13(a)-13(c), we show the profiles of the macroscopic quantities $(\mathcal{D})_{\mathrm{R}},\left(\mathcal{V}_{r}\right)_{\mathrm{R}},\left(\mathcal{V}_{\theta}\right)_{\mathrm{R}}$, $\left(\mathcal{V}_{z}\right)_{\mathrm{I}}$, and $(\mathcal{T})_{\mathrm{R}}$ pertaining to the eigenfunction $\mathcal{F}$ at three points on the neutral stability curve for $\alpha=\pi$ (solid curve) in Fig. 12, i.e., $\left(V_{I I} /\left(2 R T_{0}\right)^{1 / 2}, V_{I} /\left(2 R T_{0}\right)^{1 / 2}\right)=(-0.6,0.741)$ $[(\mathrm{a})],(0,0.397)[(\mathrm{b})]$, and $(0.3,0.903)[(\mathrm{c})]$. In the right-hand figures of Figs. 13(a)-13(c), we show the corresponding flow velocity vector of the disturbance $\left(\left(\mathcal{V}_{r}\right)_{\mathrm{R}} \cos \alpha \hat{z}\right.$, $\left.-\left(\mathcal{V}_{z}\right)_{\mathrm{I}} \sin \alpha \hat{z}\right)$ in the $\left(r / L_{I}, z / L_{I}\right)$ plane. Figure 14 contains corresponding figures at three points on the neutral stability curve for $\alpha=2 \pi$ (solid curve) in Fig. 12, i.e., $\left(V_{I I} /\left(2 R T_{0}\right)^{1 / 2}, V_{I} /\left(2 R T_{0}\right)^{1 / 2}\right)=(-0.6,0.771)[(\mathrm{a})],(0,0.623)$ $[(\mathrm{b})]$, and $(0.3,1.196)[(\mathrm{c})]$. In the square domain $L_{I} \leqslant r$ $\leqslant L_{I I}=2 L_{I}$ and $0 \leqslant z \leqslant L_{z}=L_{I}$, the disturbance at the neutral stability point is of single-vortex type in Figs. 13(b) and 13(c) and of double-vortex type (with two vortices arranged in the $z$ direction) in Figs. 14(b) and 14(c). In contrast, it shows a different structure in the case of Figs. 13(a) and 14(a). In Fig. 13(a), in addition to the main vortex, there appears a weak vortex near the outer cylinder. In Fig. 14(a), we observe a four-vortex structure with two main vortices arranged in the $z$ direction and two weak vortices appearing near the outer cylinder. As in Fig. 9, the quantities in the left-hand figures in Figs. 13 and 14 are normalized in such a

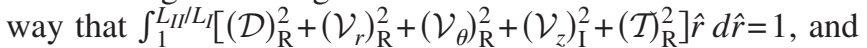
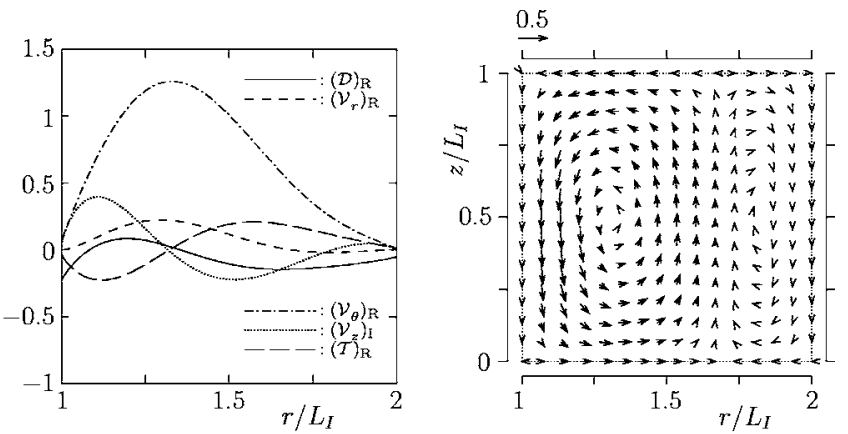

(a)
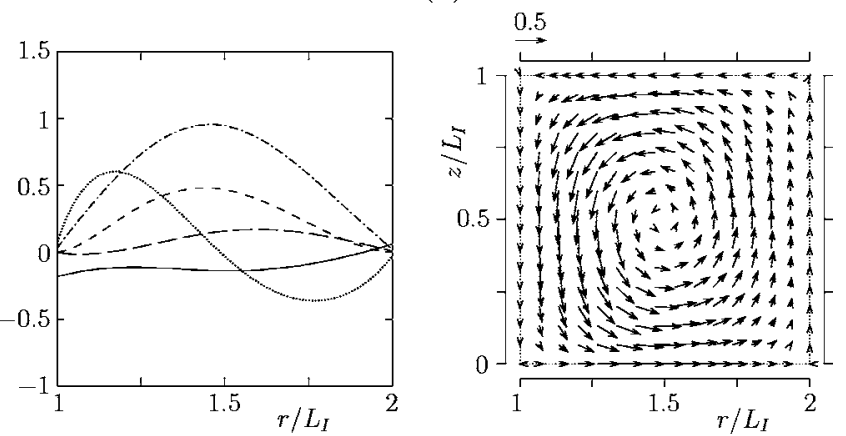

(b)
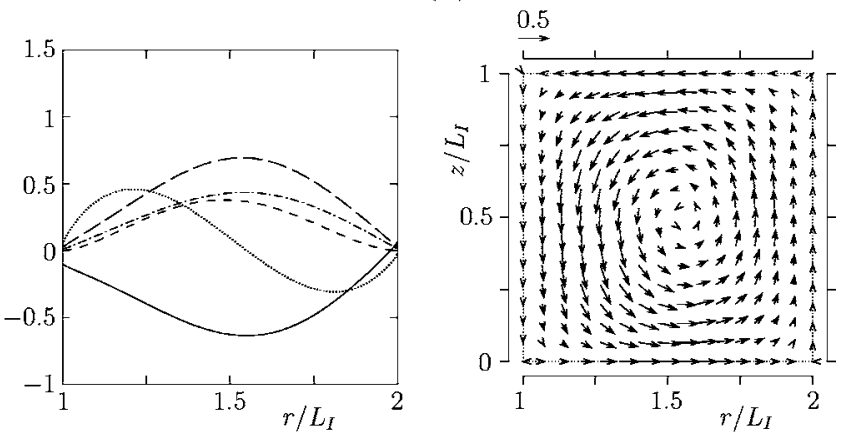

(c)

FIG. 13. Macroscopic quantities pertaining to $\mathcal{F}$ at three neutral stability points for $\alpha=\pi$ at $\mathrm{Kn}=0.0127 \quad\left(L_{I I} / L_{I}=2\right)$. $\left(V_{I I} /\left(2 R T_{0}\right)^{1 / 2}, V_{I} /\left(2 R T_{0}\right)^{1 / 2}\right)=$ (a) $(-0.6,0.741)$, (b) $(0,0.397)$, and (c) $(0.3,0.903)$. See the caption of Fig. 9 .

the scale based on this normalization is shown in their righthand figures.

Let us go back to Fig. 12. For $V_{I I} /\left(2 R T_{0}\right)^{1 / 2} \leqq 0.3$, the neutral stability curve for the BGK model for $\alpha=\pi$ lies more or less between the symbols and $\bigcirc$, and that for $\alpha=2 \pi$ lies below (or almost on) the symbols $\boldsymbol{\nabla}$. They are also not very far from the corresponding curves (dashed lines) based on the CNS system with hard-sphere viscosity and thermal conductivity. For larger $V_{I I} /\left(2 R T_{0}\right)^{1 / 2}$, however, the discrepancy between the neutral stability curves for the BGK model and those based on the CNS system for hard-sphere molecules becomes significant. The former curves are also inconsistent with the DSMC result at $V_{I I} /\left(2 R T_{0}\right)^{1 / 2}=0.6$. This discrepancy is also due to the fact that the conversion of the Knudsen number (27) is not appropriate for high-speed rotation of the cylinders. The neutral stability curve for the BGK model for $\alpha=2 \pi$ crosses that for $\alpha=\pi$ at $V_{I I} /\left(2 R T_{0}\right)^{1 / 2}$ 

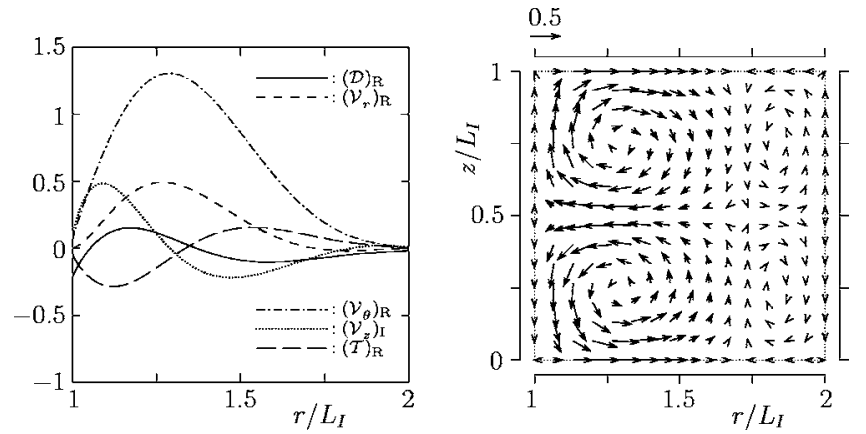

(a)
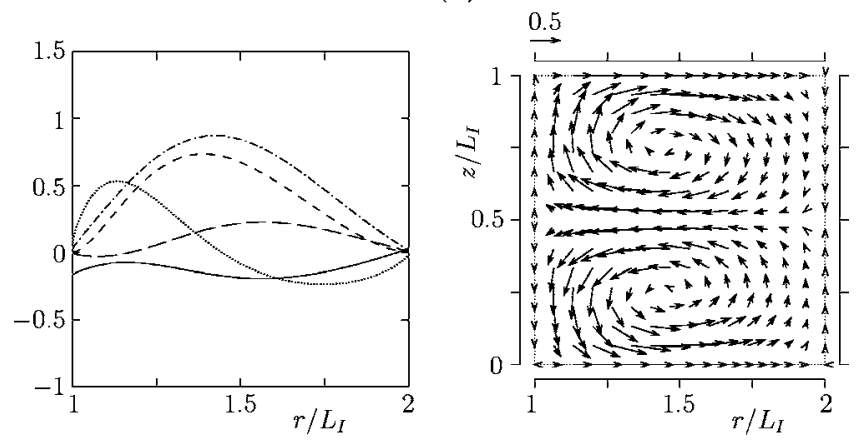

(b)
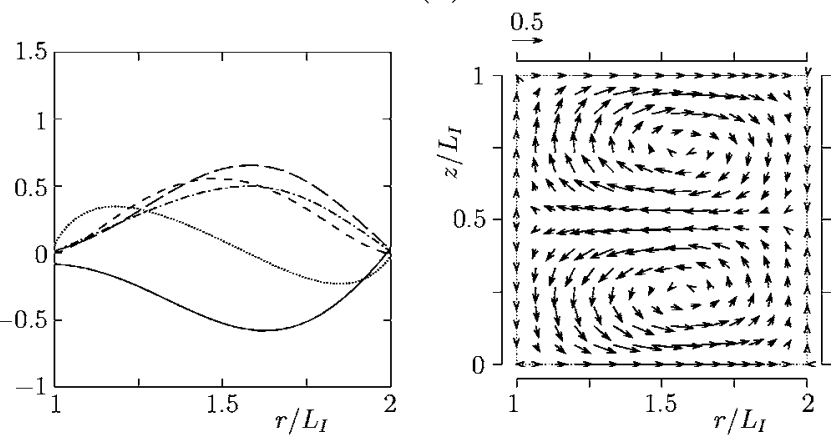

(c)

FIG. 14. Macroscopic quantities pertaining to $\mathcal{F}$ at three neutral stability points for $\alpha=2 \pi$ at $\mathrm{Kn}=0.0127 \quad\left(L_{I I} / L_{I}=2\right)$. $\left(V_{I I} /\left(2 R T_{0}\right)^{1 / 2}, V_{I} /\left(2 R T_{0}\right)^{1 / 2}\right)=($ a) $(-0.6,0.771)$, (b) $(0,0.623)$, and (c) $(0.3,1.196)$. See the caption of Fig. 9.

$=-0.746$ and goes slightly below for smaller $V_{I I} /\left(2 R T_{0}\right)^{1 / 2}$ though it is not clearly visible in the figure. This feature is seen more clearly for the neutral stability curves based on the CNS system (hard-sphere molecules). This means that, for a fixed $V_{I I} /\left(2 R T_{0}\right)^{1 / 2}$ in this zone, as $V_{I} /\left(2 R T_{0}\right)^{1 / 2}$ increases, the cylindrical Couette flow first becomes unstable to the disturbance that may induce a double-vortex flow. This tendency is observed in the DSMC result for slightly larger values of $V_{I I} /\left(2 R T_{0}\right)^{1 / 2}$. That is, at $V_{I I} /\left(2 R T_{0}\right)^{1 / 2}=-0.6$ (see Fig. 5), the region of a cylindrical Couette flow $(O)$ is in contact with the region of a double-vortex flow $(\boldsymbol{\nabla})$, and we are not able to obtain a stable single-vortex flow around there.

\section{Computational system and accuracy}

In this section, we summarize the computational systems used in the finite-difference analysis in the present study and some accuracy tests.
In the computation for the cylindrical Couette flow in Sec. III B 1 , the region $1 \leqslant \hat{r} \leqslant L_{I I} / L_{I}$ is divided into $N$ nonuniform intervals, the smallest intervals being at the cylinders and the largest around the middle of the gap between the cylinders; the region $0 \leqslant \zeta<\infty$ is replaced by a finite interval $0 \leqslant \zeta \leqslant \zeta_{D}$ and then divided into $M_{1}$ nonuniform intervals, the smallest intervals being around $\zeta=0$ and the largest at $\zeta=\zeta_{D}$; the region $-\pi \leqslant \theta_{\zeta} \leqslant \pi$ is divided into $M_{2}$ uniform intervals. It is noted that $\zeta_{z}$ can be eliminated by a suitable transformation in the present axially and circumferentially uniform case $[9,37]$. We use the following three systems: $\left(N, \zeta_{D}, M_{1}, M_{2}\right)=(120,6,48,272) \quad($ system $A)$ for $\hat{V}_{I}<1.5$; $(120,8.167,56,544) \quad($ system $B)$ for $1.5 \leqslant \hat{V}_{I}<3$; and $(240,9.375,60,1088)$ (system $C$ ) for $3 \leqslant \hat{V}_{I}$. The smallest and largest intervals $\Delta \hat{r}_{s}$ and $\Delta \hat{r}_{l}$ in $\hat{r}$ and the smallest and largest intervals $\Delta \zeta_{s}$ and $\Delta \zeta_{l}$ in $\zeta$ in these systems are as follows: $\quad\left(\Delta \hat{r}_{s}, \Delta \hat{r}_{l} ; \Delta \zeta_{s}, \Delta \zeta_{l}\right)=\left(1.713 \times 10^{-4}, 1.309 \times 10^{-2}\right.$; $\left.2.604 \times 10^{-3}, 0.2474\right) \quad($ system $\quad A), \quad\left(1.713 \times 10^{-4}, 1.309\right.$ $\left.\times 10^{-2} ; 2.604 \times 10^{-3}, 0.2891\right) \quad($ system $B)$, and $(4.284$ $\left.\times 10^{-5}, 6.545 \times 10^{-3} ; 2.604 \times 10^{-3}, 0.3099\right)$ (system $\left.C\right)$.

In the computation of Eqs. (15a)-(15c) in Sec. III B 2, the way of discretization for $\hat{r}, \zeta$, and $\theta_{\zeta}$ is the same as that for the cylindrical Couette flow. As for $\zeta_{z}$, we consider the half region $\zeta_{z} \geqslant 0$ taking into account the property (21); then it is replaced by a finite region $0 \leqslant \zeta_{z} \leqslant \zeta_{z D}$ and divided into $M_{3}$ nonuniform intervals, the smallest interval being around $\zeta_{z}$ $=0$ and the largest at $\zeta_{z}=\zeta_{z D}$. We used the following three systems: $\quad\left(N, \zeta_{D}, M_{1}, M_{2}, \zeta_{z D}, M_{3}\right)=(60,6,24,136,6,24)$ (system $A^{\prime}$ ) for $\hat{V}_{I}<1.5,(120,8,36,272,6,24)$ (system $B^{\prime}$ ) for $1.5 \leqslant \hat{V}_{I}<3$, and $(120,10,48,272,6,24)$ (system $C^{\prime}$ ) for $3 \leqslant \hat{V}_{I}$. The dimensionless time step $\Delta \hat{t}$ is taken to be $\Delta \hat{t}$ $=\mathrm{Kn} / 5$, which means that the dimensional time step is about $22 \%$ of the mean free time of the gas. Let $\Delta \zeta_{z s}$ and $\Delta \zeta_{z l}$ be the smallest and largest intervals in $\zeta_{z}$. Then, the smallest and largest intervals in these systems are as follows: $\quad\left(\Delta \hat{r}_{s}, \Delta \hat{r}_{l} ; \Delta \zeta_{s}, \Delta \zeta_{l} ; \Delta \zeta_{z s}, \Delta \zeta_{z l}\right)=\left(6.852 \times 10^{-4}, 2.617\right.$ $\left.\times 10^{-2} ; 1.042 \times 10^{-2}, 0.4896 ; 1.042 \times 10^{-2}, 0.4896\right) \quad$ (system $\left.A^{\prime}\right), \quad\left(1.713 \times 10^{-4}, 1.309 \times 10^{-2} ; 6.173 \times 10^{-3}, 0.4383 ; 1.042\right.$ $\left.\times 10^{-2}, 0.4896\right) \quad\left(\right.$ system $\left.B^{\prime}\right), \quad$ and $\left(1.713 \times 10^{-4}, 1.309\right.$ $\times 10^{-2} ; 4.340 \times 10^{-3}, 0.4124 ; 1.042 \times 10^{-2}, 0.4896$ ) (system $\left.C^{\prime}\right)$.

Since the computation of the system (15a)-(15c) is rather heavy, it is not easy to perform a systematic and thorough check of accuracy of the computation. Therefore, we have restricted ourselves to an accuracy test for the results of neutral stability points. Let us consider the neutral stability points for $\alpha=\pi$ and $\mathrm{Kn}=0.0127$ shown in Fig. 11 and Table I. In the last paragraph of Sec. III B 2, we have explained the way of obtaining a neutral stability point. That is, for a fixed value of $\hat{V}_{I I}$, we obtain $\sigma_{M}$ at some values of $\hat{V}_{I}$ in an interval that seems to contain the $\hat{V}_{I}$ corresponding to $\sigma_{M}=0$. Then, we interpolate these $\sigma_{M}$ 's using the natural cubic spline curve to obtain $\sigma_{M}$ as a function of $\hat{V}_{I}: \sigma_{M}=S\left(\hat{V}_{I}\right)$. Thus, the neutral stability point for the $\hat{V}_{I I}$ is obtained as $\hat{V}_{I}=S^{-1}(0)$. In the region where the gradient of the neutral stability curve is large, the roles of $\hat{V}_{I I}$ and $\hat{V}_{I}$ are interchanged. 
We now consider the three points $P_{1}\left(\hat{V}_{I I}, \hat{V}_{I}\right)=(0,0.397)$, $P_{2}(0.3,0.903)$, and $P_{3}(0.423,2)$ on the neutral stability curve for $\mathrm{Kn}=0.0127$ in Fig. 11 (see Table I). The point $P_{1}$ is obtained by spline interpolation based on the four points $\left(\hat{V}_{I I}, \hat{V}_{I}\right)=(0,0.3),(0,0.4),(0,0.5)$, and $(0,0.6)$; the point $P_{2}$ from that based on $\left(\hat{V}_{I I}, \hat{V}_{I}\right)=(0.3,0.8),(0.3,0.9),(0.3,1.0)$, and $(0.3,1.1)$; the point $P_{3}$ from that based on $\left(\hat{V}_{I I}, \hat{V}_{I}\right)$ $=(0.40,2),(0.41,2),(0.42,2)$, and $(0.43,2)$. The system $(A$ $+A^{\prime}$ ) is used for the points $P_{1}$ and $P_{2}$ (that is, system $A$ is used for the cylindrical Couette flow, and system $A^{\prime}$ for the linear-stability analysis), whereas the system $\left(B+B^{\prime}\right)$ is used for the point $P_{3}$. First, in order to check if the spline interpolation gives a correct value of $\hat{V}_{I}$ or $\hat{V}_{I I}$ corresponding to $\sigma_{M}=0$, we compute $\sigma_{M}$ at points close to $P_{1}, P_{2}$, and $P_{3}$, i.e., $P_{1}^{ \pm}\left(\hat{V}_{I I}, \hat{V}_{I}\right)=(0,0.397 \pm 0.001), P_{2}^{ \pm}(0.3,0.903 \pm 0.001)$, and $P_{3}^{ \pm}(0.423 \pm 0.001,2)$. Then we obtain $\sigma_{M}=-0.3164 \times 10^{-3}$ at $P_{1}^{-}$and $\sigma_{M}=0.3792 \times 10^{-3}$ at $P_{1}^{+} ; \sigma_{M}=-0.4805 \times 10^{-3}$ at $P_{2}^{-}$ and $\sigma_{M}=0.2647 \times 10^{-3}$ at $P_{2}^{+}$; and $\sigma_{M}=0.3174 \times 10^{-2}$ at $P_{3}^{-}$ and $\sigma_{M}=-0.1689 \times 10^{-2}$ at $P_{3}^{+}$. From these values, it is seen that the spline interpolation gives the value of $\hat{V}_{I}$ or $\hat{V}_{I I}$ corresponding to $\sigma_{M}=0$ with an error smaller than \pm 0.001 . Next, we compute the neutral stability points corresponding to $P_{1}, P_{2}$, and $P_{3}$ with different lattice systems, that is, the finer system $\left(B+B^{\prime}\right)$ for $P_{1}$ and $P_{2}$, and the coarser system $\left(A+A^{\prime}\right)$ for $P_{3}$. The result is $\left(\hat{V}_{I I}, \hat{V}_{I}\right)=(0,0.396)$ for $P_{1}$, $(0.3,0.900)$ for $P_{2}$, and $(0.425,2)$ for $P_{3}$. The difference is smaller than $0.5 \%$.

The computation has been performed mainly on CRAY Origin 2000 computers in the Institute for Chemical Research, Kyoto University and PC's with Pentium IV processors $(2.4 / 3.0 \mathrm{GHz})$ in the laboratory of Molecular Fluid Dynamics, Department of Mechanical Engineering and Science, Kyoto University.

\section{CONCLUDING REMARKS}

In the present study, we considered rarefied gas flows between two coaxial circular cylinders rotating at different angular velocities. The stability of the cylindrical Couette flow (a flow with axial and circumferential uniformity) for small disturbances was investigated by means of a linear-stability analysis. Such an analysis for the cylindrical Couette flow, which is classical in continuum fluid dynamics, has not been attempted, to the authors' knowledge, for a rarefied gas so far because of the complexity of the basic kinetic equations. In the present study, we have performed such an analysis on the basis of the BGK model of the Boltzmann equation, and for a certain ratio of the radii of the cylinders $\left(L_{I I} / L_{I}=2\right)$, we have investigated the stability of the cylindrical Couette flow for disturbances with circumferential uniformity and with certain periodicity in the axial direction $(\alpha=\pi$ and $2 \pi)$. As a result, we were able to obtain the corresponding neutral stability curves, the boundary between the stable and unstable zones for the cylindrical Couette flow, in the parameter planes. The results were compared with the corresponding neutral stability curves based on continuum models. The re- sults based on the compressible Navier-Stokes equations with the BGK viscosity and thermal conductivity, in general, show good agreement with those based on the BGK model. However, the discrepancy becomes significant when the outer cylinder is rotating at fast rotation speed in the same direction as the inner. This is due to the fact that the local Knudsen number near the inner cylinder becomes larger than the overall Knudsen number Kn because of the density drop caused by the strong centrifugal force. In addition, comparison was made with the results of numerical analysis of the flow between the two cylinders by the DSMC method for hard-sphere molecules that were obtained in our previous papers as well as in the present study. Since there is no systematic way to compare the result for the BGK model with that for hard-sphere molecules, we adopted the conventional conversion of the Knudsen number based on the assumption that the viscosity is common to both molecular models. In this comparison, the neutral stability curves based on the compressible Navier-Stokes system with hard-sphere viscosity and thermal conductivity were used as a reference. Except in the case of very high-speed rotation of the cylinders, the BGK result shows reasonable agreement with the DSMC result. A study of the effect of the molecular model on the stability of the cylindrical Couette flow in the highspeed rotation case would be an interesting subject for both the DSMC and continuum approaches.

\section{ACKNOWLEDGMENTS}

This work is supported by a Grant-in-Aid for Scientific Research (No. 17360041 and No. 16001161) from the Japan Society for the Promotion of Science and by the Center of Excellence for Research and Education on Complex Functional Mechanical Systems. The junior author (H.Y.) thanks Professor Shigeru Takata for his advice on numerical analysis.

\section{APPENDIX: CONTINUUM MODELS}

In Sec. V the results of the linear-stability analysis based on continuum models are shown for comparison. In this appendix, we specify the continuum models.

\section{Incompressible Navier-Stokes-type system}

We first consider the continuity equation and the NavierStokes equation for an incompressible fluid with the viscosity related to the mean free path as

$$
\mu=\frac{\sqrt{\pi}}{2} \frac{R \rho_{0} T_{0} \ell_{0}}{\left(2 R T_{0}\right)^{1 / 2}} \gamma_{1}
$$

where $\gamma_{1}$ is a constant depending on the molecular model, for example, $\gamma_{1}=1$ for the BGK model and 1.270042 for hard-sphere molecules. This system, together with the corresponding energy equation, and the so-called no-slip boundary condition have been derived rigorously from the Boltzmann equation and its boundary condition by a systematic asymptotic analysis for small Knudsen numbers $[33,41]$ under the condition that the flow Mach number and the relative 
temperature variation are as small as the Knudsen number. Since the velocity and pressure fields are determined only by the continuity and momentum equations, we do not consider the energy equation. The dimensionless form of the relevant equations is given by

$$
\begin{gathered}
\frac{1}{\hat{r}} \frac{\partial}{\partial \hat{r}}\left(\hat{r} \hat{v}_{r}\right)+\frac{\partial \hat{v}_{z}}{\partial \hat{z}}=0, \\
\frac{\partial \hat{v}_{r}}{\partial \hat{t}}+\hat{v}_{r} \frac{\partial \hat{v}_{r}}{\partial \hat{r}}+\hat{v}_{z} \frac{\partial \hat{v}_{r}}{\partial \hat{z}}-\frac{\hat{v}_{\theta}^{2}}{\hat{r}}=-\frac{1}{2} \frac{\partial \hat{p}}{\partial \hat{r}}+\frac{\sqrt{\pi} \gamma_{1} \mathrm{Kn}}{4}\left(\hat{\Delta} \hat{v}_{r}-\frac{\hat{v}_{r}}{\hat{r}^{2}}\right), \\
\frac{\partial \hat{v}_{\theta}}{\partial \hat{t}}+\hat{v}_{r} \frac{\partial \hat{v}_{\theta}}{\partial \hat{r}}+\hat{v}_{z} \frac{\partial \hat{v}_{\theta}}{\partial \hat{z}}+\frac{\hat{v}_{r} \hat{v}_{\theta}}{\hat{r}}=\frac{\sqrt{\pi} \gamma_{1} \mathrm{Kn}}{4}\left(\hat{\Delta}_{\hat{v}_{\theta}}-\frac{\hat{v}_{\theta}}{\hat{r}^{2}}\right), \\
\frac{\partial \hat{v}_{z}}{\partial \hat{t}}+\hat{v}_{r} \frac{\partial \hat{v}_{z}}{\partial \hat{r}}+\hat{v}_{z} \frac{\partial \hat{v}_{z}}{\partial \hat{z}}=-\frac{1}{2} \frac{\partial \hat{p}}{\partial \hat{z}}+\frac{\sqrt{\pi} \gamma_{1} \mathrm{Kn}}{4} \hat{\Delta} \hat{v}_{z},
\end{gathered}
$$

where

$$
\hat{\Delta} \equiv \frac{\partial^{2}}{\partial \hat{r}^{2}}+\frac{1}{\hat{r}} \frac{\partial}{\partial \hat{r}}+\frac{\partial^{2}}{\partial \hat{z}^{2}},
$$

and $\hat{p}$ is the dimensionless pressure related to the dimensional pressure $p$ as $\hat{p}=p / R \rho_{0} T_{0}$. The no-slip boundary conditions for Eqs. (A2)-(A5) are

$$
\begin{gathered}
\hat{v}_{r}=\hat{v}_{z}=0, \quad \hat{v}_{\theta}=\hat{V}_{I}, \quad \text { at } \hat{r}=1, \\
\hat{v}_{r}=\hat{v}_{z}=0, \quad \hat{v}_{\theta}=\hat{V}_{I I}, \quad \text { at } \hat{r}=L_{I I} / L_{I} .
\end{gathered}
$$

The Knudsen number enters this system through the viscosity.

The linear-stability analysis of the above system is essentially the same as the classical one (e.g., [18-20]). Here, we let

$$
\begin{gathered}
\hat{v}_{r}=\epsilon \mathcal{V}_{r}(\hat{r}) \exp (s \hat{t}) \exp (i \alpha \hat{z}), \\
\hat{v}_{\theta}=\hat{v}_{\theta C}(\hat{r})+\epsilon \mathcal{V}_{\theta}(\hat{r}) \exp (s \hat{t}) \exp (i \alpha \hat{z}), \\
\hat{v}_{z}=\epsilon \mathcal{V}_{z}(\hat{r}) \exp (s \hat{t}) \exp (i \alpha \hat{z}), \\
\hat{p}=\hat{p}_{C}(\hat{r})+\epsilon \mathcal{P}(\hat{r}) \exp (s \hat{t}) \exp (i \alpha \hat{z}),
\end{gathered}
$$

where $\hat{v}_{\theta C}$ and $\hat{p}_{C}$ are the velocity ( $\theta$ component) and pressure of the cylindrical Couette flow solution, $\epsilon(>0)$ is a small parameter, $\alpha$ is a real number, $s$ is a complex number, and $\mathcal{V}_{r}, \mathcal{V}_{\theta}, \mathcal{V}_{z}$, and $\mathcal{P}$ are complex-valued functions of $\hat{r}$. If we insert Eqs. (A9a)-(A9d) into Eqs. (A2)-(A8) and neglect the products of $\epsilon$, we obtain an eigenvalue problem for $\mathcal{V}_{r}$, $\mathcal{V}_{\theta}, \mathcal{V}_{z}, \mathcal{P}$, and $s$. The problem is essentially the same as that appearing in the first-order bifurcation analysis in Ref. [42], where the effect of evaporation and condensation on the bifurcation of the flow between two coaxial rotating cylinders is investigated. We have exploited the solution method of Ref. [42] to solve the eigenvalue problem, which led to the results contained in Figs. 7, 8, and 11. It should be mentioned that a detailed bifurcation analysis of the flow between two rotating cylinders has been performed in Ref. [31], in the case where the speed of rotation of the cylinders is low but the temperature difference of the cylinders is large, with special interest in the ghost effect on the temperature field caused by the infinitesimal Taylor-vortex flow in the continuum limit. The above eigenvalue problem is contained in the first-order bifurcation analysis there, as the special case where the temperatures of the two cylinders are the same.

\section{Compressible Navier-Stokes-type system}

Next, we consider the Navier-Stokes system for a compressible fluid with the following formulas of viscosity and thermal conductivity:

$$
\mu=\frac{\sqrt{\pi}}{2} \frac{R \rho_{0} T_{0} \ell_{0}}{\left(2 R T_{0}\right)^{1 / 2}} \hat{\gamma}_{1} \hat{T}^{1 / 2},
$$

$$
\lambda=\frac{5 \sqrt{\pi}}{4} \frac{R^{2} \rho_{0} T_{0} \ell_{0}}{\left(2 R T_{0}\right)^{1 / 2}} \hat{\gamma}_{2} \hat{T}^{1 / 2},
$$

where $\hat{\gamma}_{1}$ and $\hat{\gamma}_{2}$ are functions of $\hat{T}$, and their functional form depends on the molecular model, for example, $\hat{\gamma}_{1}=\hat{\gamma}_{2}=\sqrt{\hat{T}}$ for the BGK model, and $\hat{\gamma}_{1}=1.270042$ and $\hat{\gamma}_{2}=1.922284$ for hard-sphere molecules. These formulas are obtained by the systematic asymptotic analysis of the Boltzmann equation $[33,43]$. The dimensionless form of the continuity, momentum, and energy equations as well as the equation of state are written as follows:

$$
\frac{\partial \hat{\rho}}{\partial \hat{t}}+\frac{1}{\hat{r}} \frac{\partial}{\partial \hat{r}}\left(\hat{r} \hat{\rho} \hat{v}_{r}\right)+\frac{\partial}{\partial \hat{z}}\left(\hat{\rho} \hat{v}_{z}\right)=0,
$$

$$
\begin{gathered}
\hat{\rho}\left(\frac{\partial \hat{v}_{r}}{\partial \hat{t}}+\hat{v}_{r} \frac{\partial \hat{v}_{r}}{\partial \hat{r}}-\frac{\hat{v}_{\theta}^{2}}{\hat{r}}+\hat{v}_{z} \frac{\partial \hat{v}_{r}}{\partial \hat{z}}\right) \\
=-\frac{1}{2} \frac{\partial \hat{p}^{*}}{\partial \hat{r}}+\frac{\sqrt{\pi} \mathrm{Kn}}{4}\left\{\frac{2}{\hat{r}} \frac{\partial}{\partial \hat{r}}\left(\hat{\gamma}_{1} \hat{T}^{1 / 2} \hat{r} \frac{\partial \hat{v}_{r}}{\partial \hat{r}}\right)-2 \hat{\gamma}_{1} \hat{T}^{1 / 2} \frac{\hat{v}_{r}}{\hat{r}^{2}}\right. \\
\left.+\frac{\partial}{\partial \hat{z}}\left[\hat{\gamma}_{1} \hat{T}^{1 / 2}\left(\frac{\partial \hat{v}_{r}}{\partial \hat{z}}+\frac{\partial \hat{v}_{z}}{\partial \hat{r}}\right)\right]\right\}, \\
\hat{\rho}\left(\frac{\partial \hat{v}_{\theta}}{\partial \hat{t}}+\hat{v}_{r} \frac{\partial \hat{v}_{\theta}}{\partial \hat{r}}+\frac{\hat{v}_{r} \hat{v}_{\theta}}{\hat{r}}+\hat{v}_{z} \frac{\partial \hat{v}_{\theta}}{\partial \hat{z}}\right) \\
=\frac{\sqrt{\pi} \operatorname{Kn}}{4}\left\{\frac{1}{\hat{r}^{2}} \frac{\partial}{\partial \hat{r}}\left[\hat{\gamma}_{1} \hat{T}^{1 / 2} \hat{r}^{3} \frac{\partial}{\partial \hat{r}}\left(\frac{\hat{v}_{\theta}}{\hat{r}}\right)\right]+\frac{\partial}{\partial \hat{z}}\left(\hat{\gamma}_{1} \hat{T}^{1 / 2} \frac{\partial \hat{v}_{\theta}}{\partial \hat{z}}\right)\right\},
\end{gathered}
$$




$$
\begin{aligned}
& \hat{\rho}\left(\frac{\partial \hat{v}_{z}}{\partial \hat{t}}+\hat{v}_{r} \frac{\partial \hat{v}_{z}}{\partial \hat{r}}+\hat{v}_{z} \frac{\partial \hat{v}_{z}}{\partial \hat{z}}\right) \\
& =-\frac{1}{2} \frac{\partial \hat{p}^{*}}{\partial \hat{z}}+\frac{\sqrt{\pi} \mathrm{Kn}}{4}\left\{\frac{1}{\hat{r}} \frac{\partial}{\partial \hat{r}}\left[\hat{\gamma}_{1} \hat{T}^{1 / 2} \hat{r}\left(\frac{\partial \hat{v}_{r}}{\partial \hat{z}}+\frac{\partial \hat{v}_{z}}{\partial \hat{r}}\right)\right]\right. \\
& \left.+2 \frac{\partial}{\partial \hat{z}}\left(\hat{\gamma}_{1} \hat{T}^{1 / 2} \frac{\partial \hat{v}_{z}}{\partial \hat{z}}\right)\right\} \\
& \frac{3}{2} \hat{\rho}\left(\frac{\partial \hat{T}}{\partial \hat{t}}+\hat{v}_{r} \frac{\partial \hat{T}}{\partial \hat{r}}+\hat{v}_{z} \frac{\partial \hat{T}}{\partial \hat{z}}\right) \\
& =-\hat{p}^{*}\left(\frac{\partial \hat{v}_{r}}{\partial \hat{r}}+\frac{\hat{v}_{r}}{\hat{r}}+\frac{\partial \hat{v}_{z}}{\partial \hat{z}}\right) \\
& +\frac{\sqrt{\pi} \mathrm{Kn}}{4}\left\{4 \hat { \gamma } _ { 1 } \hat { T } ^ { 1 / 2 } \left[\left(\frac{\partial \hat{v}_{r}}{\partial \hat{r}}\right)^{2}+\left(\frac{\hat{v}_{r}}{\hat{r}}\right)^{2}+\left(\frac{\partial \hat{v}_{z}}{\partial \hat{z}}\right)^{2}\right.\right. \\
& \left.+\frac{1}{2}\left(\frac{\partial \hat{v}_{\theta}}{\partial \hat{r}}-\frac{\hat{v}_{\theta}}{\hat{r}}\right)^{2}+\frac{1}{2}\left(\frac{\partial \hat{v}_{r}}{\partial \hat{z}}+\frac{\partial \hat{v}_{z}}{\partial \hat{r}}\right)^{2}+\frac{1}{2}\left(\frac{\partial \hat{v}_{\theta}}{\partial \hat{z}}\right)^{2}\right] \\
& \left.+\frac{5}{2}\left[\frac{1}{\hat{r}} \frac{\partial}{\partial \hat{r}}\left(\hat{\gamma}_{2} \hat{T}^{1 / 2} \hat{r} \frac{\partial T}{\partial \hat{r}}\right)+\frac{\partial}{\partial \hat{z}}\left(\hat{\gamma}_{2} \hat{T}^{1 / 2} \frac{\partial \hat{T}}{\partial \hat{z}}\right)\right]\right\} \text {, } \\
& \hat{p}=\hat{\rho} \hat{T},
\end{aligned}
$$

where

$$
\hat{p}^{*}=\hat{p}+\frac{\sqrt{\pi}}{3} \operatorname{Kn} \hat{\gamma}_{1} \hat{T}^{1 / 2}\left(\frac{\partial \hat{v}_{r}}{\partial \hat{r}}+\frac{\hat{v}_{r}}{\hat{r}}+\frac{\partial \hat{v}_{z}}{\partial \hat{z}}\right) .
$$

The no-slip boundary conditions at the cylinders are

$$
\begin{gathered}
\hat{v}_{r}=\hat{v}_{z}=0, \quad \hat{v}_{\theta}=\hat{V}_{I}, \quad \hat{T}=1, \quad \text { at } \hat{r}=1, \\
\hat{v}_{r}=\hat{v}_{z}=0, \quad \hat{v}_{\theta}=\hat{V}_{I I}, \quad \hat{T}=1, \quad \text { at } \hat{r}=L_{I I} / L_{I} .
\end{gathered}
$$

The Knudsen number enters the above system through the viscosity and thermal conductivity.

The linear stability of the cylindrical Couette flow has been investigated on the basis of the compressible NavierStokes system in Refs. [44,45]. The system used in these references is essentially the same as the above system. Here, we let

$$
\begin{aligned}
& \hat{\rho}=\hat{\rho}_{C}(\hat{r})+\epsilon \mathcal{D}(\hat{r}) \exp (s \hat{t}) \exp (i \alpha \hat{z}), \\
& \hat{T}=\hat{T}_{C}(\hat{r})+\epsilon \mathcal{T}(\hat{r}) \exp (s \hat{t}) \exp (i \alpha \hat{z}),
\end{aligned}
$$

in addition to the form of Eqs. (A9a)-(A9d), where $\hat{\rho}_{C}, \hat{v}_{\theta C}$, $\hat{T}_{C}$, and $\hat{p}_{C}$ are the density, velocity ( $\theta$ component), temperature, and pressure of the cylindrical Couette flow solution of the above system, and $\mathcal{D}, \mathcal{V}_{r}, \mathcal{V}_{\theta}, \mathcal{V}_{z}, \mathcal{T}$, and $\mathcal{P}$ are complexvalued functions of $\hat{r}$. We derive the boundary-value problem of the system of linear ordinary differential equations for the disturbances $\mathcal{D}, \mathcal{V}_{r}, \mathcal{V}_{\theta}, \mathcal{V}_{z}, \mathcal{T}$, and $\mathcal{P}$, which forms an eigenvalue problem. We solved this problem using the same technique as in Ref. [45] to obtain the results shown in Figs. 7, 8, and $10-12$. In this case, we are able to attain much higher accuracy than in the case of the BGK system.

It should be mentioned that similar continuum equations, i.e., the equations of compressible Navier-Stokes type with viscosity and thermal conductivity of the form of Eqs. (A10a) and (A10b) (more precisely, their approximations), have been employed for the study of the Rayleigh-Bénard problem $[46,47]$.
[1] C. Cercignani and F. Sernagiotto, Phys. Fluids 10, 1200 (1967).

[2] K. Nanbu, Phys. Fluids 27, 2632 (1984).

[3] R. E. Khayat and B. C. Eu, Phys. Rev. A 38, 2492 (1988).

[4] R. E. Khayat and B. C. Eu, Phys. Rev. A 39, 728 (1989).

[5] F. Sharipov and G. Kremer, Transp. Theory Stat. Phys. 25, 217 (1996).

[6] F. Sharipov and G. Kremer, Eur. J. Mech. B/Fluids 15, 493 (1996).

[7] Y. Sone, S. Takata, and H. Sugimoto, Phys. Fluids 8, 3403 (1996).

[8] K. W. Tibbs, F. Baras, and A. L. Garcia, Phys. Rev. E 56, 2282 (1997).

[9] Y. Sone, H. Sugimoto, and K. Aoki, Phys. Fluids 11, 476 (1999).

[10] Y. Sone, T. Ohwada, and Y. Makihara, in Rarefied Gas Dynamics, edited by R. Brun, R. Campargue, R. Gatignol, and J.-C. Lengrand (Cépaduès-Éditions, Toulouse, 1999), Vol. 1, p. 511.

[11] Y. Sone and T. Doi, Phys. Fluids 12, 2639 (2000).

[12] L. M. de Socio, N. Ianiro, and L. Marino, J. Thermophys. Heat Transfer 14, 269 (2000).
[13] L. M. de Socio and L. Marino, Math. Models Meth. Appl. Sci. 10, 73 (2000).

[14] Y. Sone, M. Handa, and H. Sugimoto, Transp. Theory Stat. Phys. 31, 299 (2002).

[15] K. Aoki, H. Yoshida, T. Nakanishi, and A. L. Garcia, Phys. Rev. E 68, 016302 (2003).

[16] S. Yuhong, R. W. Barber, and D. R. Emerson, Phys. Fluids 17, 047102 (2005).

[17] G. I. Taylor, Philos. Trans. R. Soc. London, Ser. A 223, 289 (1923).

[18] S. Chandrasekhar, Hydrodynamic and Hydromagnetic Stability (Oxford University Press, London, 1961).

[19] E. M. Sparrow, W. D. Munro, and V. K. Jonsson, J. Fluid Mech. 20, 35 (1964).

[20] K. Kirchgässner and P. Sorger, Q. J. Mech. Appl. Math. 22, 183 (1969).

[21] E. L. Koschmieder, Bénard Cells and Taylor Vortices (Cambridge University Press, Cambridge, UK, 1993).

[22] P. Chossat and G. Iooss, The Couette-Taylor Problem (Springer-Verlag, New York, 1994).

[23] G. A. Bird, Molecular Gas Dynamics (Oxford University 
Press, Oxford, 1976).

[24] G. A. Bird, Molecular Gas Dynamics and the Direct Simulation of Gas Flows (Oxford University Press, Oxford, 1994).

[25] S. Stefanov and C. Cercignani, J. Fluid Mech. 256, 199 (1993).

[26] D. Reichelmann and K. Nanbu, Phys. Fluids A 5, 2585 (1993).

[27] M. Usami, in Rarefied Gas Dynamics, edited by J. Harvey and G. Lord (Oxford University Press, Oxford, 1995), p. 389.

[28] B. A. Bird, Comput. Math. Appl. 35, Nos. 1-2, 1 (1998).

[29] K. Aoki, Y. Sone, and M. Yoshimoto, in Rarefied Gas Dynamics (Ref. [10]), Vol. 2, p. 109.

[30] H. Yoshida and K. Aoki, in Rarefied Gas Dynamics, edited by M. Capitelli (AIP, Melville, NY, 2005), p. 467.

[31] Y. Sone, M. Handa, and T. Doi, Phys. Fluids 15, 2903 (2003)

[32] Y. Sone, Annu. Rev. Fluid Mech. 32, 779 (2000).

[33] Y. Sone, Kinetic Theory and Fluid Dynamics (Birkhäuser, Boston, 2002).

[34] P. L. Bhatnagar, E. P. Gross, and M. Krook, Phys. Rev. 94, 511 (1954).
[35] P. Welander, Ark. Fys. 7, 507 (1954).

[36] M. N. Kogan, J. Appl. Math. Mech. 22, 597 (1958).

[37] H. Sugimoto and Y. Sone, Phys. Fluids A 4, 419 (1992).

[38] Y. Sone and H. Sugimoto, Phys. Fluids A 5, 1491 (1993).

[39] Y. Sone and S. Takata, Transp. Theory Stat. Phys. 21, 501 (1992).

[40] R. S. Myong, Phys. Fluids 11, 2788 (1999).

[41] Y. Sone, in Rarefied Gas Dynamics, edited by D. Dini (Editrice Tecnico Scientifica, Pisa, 1971), Vol. 2, p. 737.

[42] Y. Sone and T. Doi, in Rarefied Gas Dynamics, edited by A. D. Ketsdever and E. P. Muntz (AIP, Melville, NY, 2003), p. 646.

[43] Y. Sone, C. Bardos, F. Golse, and H. Sugimoto, Eur. J. Mech. B/Fluids 19, 325 (2000).

[44] K.-H. Kao and C.-Y. Chow, Phys. Fluids A 4, 984 (1992).

[45] F. F. Hatay, S. Biringen, G. Erlebacher, and W. E. Zorumski, Phys. Fluids A 5, 393 (1993).

[46] S. Stefanov, V. Roussinov, and C. Cercignani, Phys. Fluids 14, 2270 (2002).

[47] A. Manela and I. Frankel, Phys. Fluids 17, 036101 (2005). 